\title{
Il Dialogo della volgar lingua di Pierio Valeriano (1529-1530)
}

Introduzione a un trattato cinquecentesco sul volgare italiano

\section{Maria Luisa Giordano}

\section{(2) OpenEdition}

\section{Journals}

Edizione digitale

URL: https://journals.openedition.org/eve/1135

DOI: 10.4000/eve.1135

ISSN: 2425-1593

Editore:

Université de Savoie, Université Jean Moulin - Lyon 3

Notizia bibliografica digitale

Maria Luisa Giordano, «ll Dialogo della volgar lingua di Pierio Valeriano (1529-1530)», Corpus Eve

[Online], Area italiana, online dal 03 avril 2015, consultato il 16 avril 2022. URL: http://

journals.openedition.org/eve/1135 ; DOl: https://doi.org/10.4000/eve.1135

Questo documento è stato generato automaticamente il 16 avril 2022.

(c) Tous droits réservés 


\title{
Il Dialogo della volgar lingua di Pierio Valeriano (1529-1530)
}

Introduzione a un trattato cinquecentesco sul volgare italiano

\author{
Maria Luisa Giordano
}

\section{NOTIZIA}

Dialogo della Volgar Lingua. Di Gio. Pierio Valeriano bellunese, non prima uscito in luce. All'Illustr.mo et Reverend.mo Sig. il Principe Don Carlo Cardinal de' Medici. Con licenza de' Superiori, et Privilegio, in Venetia, nella stamperia di Gio. Battista Ciotti, 1620

\section{Note sull'autore e sul contesto linguistico}

1 Giovanni Pietro Bolzani Dalle Fosse nacque a Belluno nel 1477 da una famiglia di modesta estrazione sociale ${ }^{1}$. Compì i primi studi nella città natale e grazie al sostegno dello zio Urbano Bolzanio, illustre grecista e collaboratore di Aldo Manuzio² ${ }^{2}$, fra il 1492 e il 1493 si trasferì a Venezia, dove ebbe come maestri gli umanisti Benedetto Brugnolo da Legnago, Giorgio Valla e Marco Antonio Cocci detto il Sabellico. Proprio su suggerimento del Sabellico, il Dalle Fosse assunse il nome umanistico di Pierio Valeriano. Da alcuni accenni contenuti nella sua produzione giovanile si può desumere che in quegli anni si mantenesse agli studi lavorando come precettore presso famiglie nobili e come correttore di testi, attività molto richiesta soprattutto a Venezia, la città che contava il più alto numero di tipografie. Presso lo stampatore Giovanni Tacuino pubblicò la sua opera d'esordio, una raccolta di poesie latine intitolata Praeludia quaedam (1509).

2 Per sfuggire alle guerre in cui era coinvolta Venezia, nello stesso 1509 si trasferì a Roma, uno dei principali poli d'attrazione per gli intellettuali dell'epoca, dove, grazie al sostegno di Leone X prima e di Clemente VII poi, rivestì diverse cariche prestigiose; nell'anno accademico 1521-1522 e presumibilmente nel successivo si dedicò 
all'insegnamento universitario; inoltre, durante il periodo che trascorse nell'Urbe frequentò i cenacoli umanistici ${ }^{3}$ e approntò edizioni, castigazioni e commenti di testi classici. Negli anni tra il 1524 e il 1527 soggiornò a Firenze, dove fu il precettore di Ippolito e di Alessandro de' Medici, nipoti di Clemente VII. Dal papa mecenate ottenne anche la pievania di Castion, nel Bellunese.

In seguito all'assedio e al Sacco di Roma, evento di cui non fu testimone diretto ma che lo colpì profondamente, il Valeriano maturò una visione pessimistica della condizione degli intellettuali, che lo indusse a comporre il De Litteratorum infelicitate. Nel 1537, dopo la morte di Clemente VII, Ippolito e Alessandro de' Medici, solo e deluso si ritirò a Belluno, dove poté dedicarsi all'opera per la quale raccoglieva materiali da anni, gli Hieroglyphica; inoltre, pare che nel 1540 abbia partecipato all'esperienza dell'accademia padovana degli Infiammati ${ }^{4}$. Morì a Padova nel 1558.

Il dialogo latino De Litteratorum infelicitate fu portato a termine nel 1529 ma edito solo nel 1620: è uno dei più notevoli repertori di notizie sull'Umanesimo italiano del primo Cinquecento e una significativa testimonianza delle conseguenze che gli eventi calamitosi del 1527 ebbero sul destino di molti intellettuali ${ }^{5}$. Tra le opere del Valeriano si ricordano anche le Castigationes et varietates Vergilianae lectionis (1521), le elegie latine riunite negli Amorum libri (1549) e infine gli Hieroglyphica, sive de sacris Ægyptiorum aliarumque gentium literis commentarii (Basilea, 1556) ${ }^{6}$.

Unica escursione nel campo del volgare è il Dialogo della volgar lingua, ambientato alla corte pontificia. Nei lustri a cavallo del 1500 numerosi intellettuali settentrionali (e veneti in particolare) tendevano a spostarsi verso le corti centro-settentrionali e la Roma papale, i più vivaci centri culturali d'Italia, in grado di assicurare riconoscimenti sociali e prestigiose opportunità di carriera. Tra Quattro e Cinquecento l'Urbe era diventata una città cosmopolita; la sua popolazione, di conseguenza, era per quattro quinti non indigena e durante il papato di Leone X (1513-1521) e di Clemente VII (1523-1534), entrambi della famiglia dei Medici, era cresciuta in particolare la presenza dei toscani. In questo clima di apertura e di intense relazioni culturali tra le corti, che vedeva «l'intensificarsi dei fenomeni di integrazione e di omologazione tra i vari centri italiani ${ }^{7} »$, assunse un'importanza decisiva la questione della lingua. Nell'ambiente antimunicipalistico della curia romana «si intrecciavano e si rincorrevano le opinioni linguistiche in un attivismo sperimentalistico ${ }^{8} »$ : è sufficiente ricordare, accanto al Dialogo della volgar lingua del Valeriano, il Castellano (1529) di Giangiorgio Trissino, ambientato a Castel Sant'Angelo, e il Cesano (1525) di Claudio Tolomei, dove la discussione prende avvio alla tavola del futuro cardinale Ippolito de' Medici.

\section{Presentazione del Dialogo della volgar lingua}

Non si può escludere che il Valeriano abbia iniziato a maturare un interesse per il volgare negli anni in cui frequentò la scuola del Sabellico, tra i cui allievi nel biennio 1501-1502 vi era anche il friulano Giovan Francesco Fortunio, futuro autore delle Regole della volgar lingua (1516). Del resto il maestro del Valeriano, umanista di origine sabina e di scuola romana trapiantato nel Veneto, pur non essendosi mai servito del volgare come lingua letteraria, nell'undicesima delle sue Enneades (1503 circa) non esitò a tracciare un quadro dell'Italia dialettale contemporanea, osservando in particolare che «quod Romae nuper offendebat, commercio gentium in dies magis excolitur ${ }^{9}$ " e mostrando di preferire una lingua composita, risultante dall'apporto di volgari diversi. 
Si tratta di una significativa testimonianza della curiosità degli umanisti per il volgare, ma anche di una visione della lingua parlata a Roma non molto diversa da quella che sta alla base della teoria cortigiana.

7 Il Dialogo della volgar lingua, composto, come vedremo, nel terzo decennio del '500, dovette attendere il secolo successivo per la pubblicazione: uscì postumo solo nel 1620 presso la stamperia veneziana di Giambattista Ciotti, grazie all'interessamento del vescovo bellunese Luigi Lollino, estimatore delle opere del Valeriano e proprietario di un'importante biblioteca. Nella nota A' lettori firmata dal Ciotti si legge infatti che il Dialogo «è stato raccolto quasi dalle ceneri e reliquie del suo autore, per opra di Monsignor Luigi Lollino Vescovo di Belluno». Nel $1813 \mathrm{fu}$ ripubblicato con il titolo di Dialogo sopra le lingue volgari: quale sia più conveniente di usare, in appendice alla Storia dei letterati e degli artisti del Dipartimento della Piave dell'erudito lombardo Stefano Ticozzi, il quale utilizzò un manoscritto di mano dello stesso monsignor Lollino. Rispetto all'editio princeps, la redazione pubblicata nel 1813 presenta alcune differenze, che non riguardano il contenuto del dibattito, bensì la struttura e in parte la veste linguistica ${ }^{10}$; ripropongono il testo del Ticozzi le edizioni del 1829 e del 1842, entrambe stampate a Milano.

8 Sul rapporto tra le due redazioni e sulla loro datazione si è recentemente espresso Valerio Vianello, che per il testo dell'edizione Ticozzi ha indicato come anno di partenza il 1524 e come termine ante quem la stampa delle Prose della volgar lingua di Pietro Bembo (1525). Assegna invece al biennio 1529-1530 la redazione data alle stampe nel 1620, che presuppone la conoscenza delle Prose bembiane ed è più curata dal punto di vista stilistico, più sintetica e caratterizzata da una maggiore articolazione strutturale $^{11}$. L'edizione più recente del testo, curata da Mario Pozzi e contenuta nel volume Discussioni linguistiche del Cinquecento (1988), riproduce l'editio princeps.

9 Scritto in uno stile vivace, il dialogo fornisce una testimonianza di quali fossero le opinioni in materia di lingua dei letterati che gravitavano intorno ad Angelo Colocci, il primo a rendere nota in un testo a stampa - l'Apologia delle Rime di Serafino Aquilano del 1503 - la propria posizione sulla «questione della lingua», senza tuttavia arrivare ad esporla in un'opera organica. Sostiene l'esistenza di una lingua comune italiana, che identifica con il volgare parlato presso la curia papale ${ }^{12}$.

10 Proprio il Colocci è figura di primo piano nella cornice del Dialogo ${ }^{13}$; come rileva Riccardo Drusi, a lui viene affidato «il richiamo [...] alla conversazione aristocratica e all'uso elegante degli intellettuali come a un modello primario: «Così penso far io in riferirvi questo ragionamento; attenderò a ricordarmi le cose, per quanto potrò; le parole saranno per lo più quelle che usiamo familiarmente in corte fra di noi ${ }^{14}{ }^{4}$. Adottando la tecnica della scena dentro la scena, l'autore fa precedere il dibattito vero e proprio da una cornice dialogica i cui personaggi sono, oltre al Colocci, Antonio Lelio Massimi e Antonio Marostica. Il primo racconta ai due amici di essere stato testimone di un'interessante disputa tra quattro intellettuali di spicco, due Lombardi e due Toscani: il ferrarese Antonio Tebaldeo, fermo su posizioni antitoscane e in particolare antifiorentine; il vicentino Giangiorgio Trissino, italianista moderato; il senese Claudio Tolomei, toscanista e difensore dell'autonomia del toscano dal latino; infine il nobile fiorentino Alessandro de' Pazzi, sostenitore del volgare della propria città.

11 Le coordinate cronologiche della conversazione fittizia si collocano nel periodo del papato leonino, tra il 1513 e il 1521, ma alla luce delle vicende biografiche dei personaggi si può ipotizzare una data più precisa, il 1518: in quell'anno, infatti, il 
Tolomei si recò per la prima volta a Roma, dove risiedevano anche il Bembo e il Trissino ${ }^{15}$. L'autore, tuttavia, introduce nel dialogo qualche elemento che rimanda alla data di composizione, come i riferimenti alla tragedia Dido in Carthagine di Alessandro de' Pazzi e all'Epistola de le lettere nuovamente aggiunte ne la lingua italiana del Trissino, entrambe del 1524.

Il Valeriano viene solitamente definito dalla critica un trissiniano moderato, che condivide le idee del Trissino riguardo al nome di «lingua italiana» da attribuire al volgare, pur riservando un ruolo di rilievo alla tradizione toscana ${ }^{16}$. Si discosta da questa conclusione Drusi, che preferisce annoverare l'umanista bellunese tra i sostenitori della lingua cortigiana romana: osserva infatti che il Dialogo della volgar lingua contiene evidenti riferimenti alla conversazione colta della curia papale, benché la lingua modello di cui si parla sia priva di un'esplicita determinazione geografica. La posizione del Valeriano sarebbe quindi più vicina a quella cortigiana del Colocci che a quella genericamente italiana del Trissino ${ }^{17}$.

In realtà il Valeriano, esponendo con lucidità le tesi sostenute dagli intellettuali che partecipano alla conversazione, più che prendere una posizione netta offre una vivida rappresentazione del dibattito, sempre improntato al decoro linguistico e al rispetto reciproco, rivelandosi un acuto osservatore dei costumi e delle relazioni tra uomini di cultura nella Roma medicea ${ }^{18}$. L'opera si distingue proprio per la moderazione e l'atmosfera di cordialità in cui si stempera ogni contrasto. Al di là delle sfumature di pensiero, è comunque evidente la simpatia accordata alle idee linguistiche del Trissino, al quale vengono affidate le riflessioni più corpose e significative del Dialogo, non molto diverse da quelle esposte nel Castellano ${ }^{19}$. Nella sua interpretazione delle tesi trissiniane, il Valeriano mette in evidenza soprattutto «gli elementi di equilibrato classicismo e di aristocratica stilizzazione ivi presenti, e sottace gli elementi di astratto razionalismo pure fondamentali nel Castellano ${ }^{20} \%$.

\section{Sintesi dell'opera}

Nella cornice introduttiva il Colocci racconta al Marostica di essere stato invitato a cena la sera precedente dal cardinale Giulio de' Medici e di aver preso parte ad un piacevole dibattito sul tema della lingua:

si ricercò se questa lingua volgare era nostra o d'altri, e se l'era toscana o di che paese, e se si poteva scriver in volgare altramente che con forme toscane. Poi si trattò se per lingua toscana s'intendeva solo la fiorentina, e sopra tutto qual convenisse a un galant'homo (p. 9) ${ }^{21}$.

Il Marostica, dopo essersi rammaricato per non aver assistito alla discussione, esprime il proprio fastidio per l'uso affettato di forme fiorentine arcaiche da parte di alcuni giovani intellettuali presuntuosi e saccenti:

Messer Angelo, non si può più vivere, dapoiché son usciti fuora certi soventi, certi eglino, certi uopi, certi chenti e simili strani galavroni; non posso passeggiar per Parione che vengono questi giovanotti dottarelli, barbette recitanti, e stanno ascoltando quel che ragioniamo insieme, e ci puntano negli accenti, nelle parole e nelle figure del dire, che non sono toscane, senza una compassion al mondo, ridendosi di noi, che, se ben havemo messo la barba bianca negli studi, non sapemo quello che mai non ci sognassemo d'imparare (p. 11) ${ }^{22}$. 
17 Dopo aver elogiato il Bembo, che parla «con quella simplicità naturale che s'usa fra galanthomini» (p. 13) ${ }^{23}$, i due interlocutori, raggiunti da Lelio Massimi, ritornano al tema iniziale. Il Colocci s'impegna a riferire la discussione della sera precedente, non senza aver prima fatto un rapido accenno al modo di parlare di ciascuno dei disputanti, ai quali riconosce «facundia et eleganza di dir»:

[...] il Pazzi e 'l Tolomei erano mirabili nella prontezza e velocità del parlar terso e toscano, il Trissino, molto castigato, né toscaneggiava del tutto, né teneva del venetiano, ma con grave temperamento servendosi dell'uno e dell'altro faceva una soave compositione. Il Thebaldeo si lasciava più portar dalla natura, ma alle belle cose che diceva davano molta grazia quei suo' accenti ferraresi (p. 15).

18 Osservazioni che sfociano in un elogio della lingua cortigiana parlata, usata nella comunicazione quotidiana dai colti frequentatori della corte papale, incluso il Bembo: «un galanthomo che, ancorché sappia della lingua toscana quel che se ne può sapere, non l'usa però se non con Fiorentini, e modestamente; con noi altri usa della lingua cortigiana» (p. 15) 24 . Le quattro qualità possedute dalla lingua cortigiana la rendono superiore alla toscana: oltre ad essere dolce e forbita, essa è comprensibile a tutti (intelligibile) - proprio in quanto strumento di comunicazione tra parlanti di origini diverse - e copiosa, cioè dotata di un ampio vocabolario.

19 A questo punto si conclude la cornice e ha inizio il resoconto della disputa: su invito del cardinale Giulio II, i commensali discutono dei propri impegni e interessi intellettuali; il Trissino, in particolare, riferisce di un'opera relativa all'aggiunta di alcune lettere all'alfabeto latino (l'Epistola de le lettere nuovamente aggiunte ne la lingua italiana), argomento che offre agli interlocutori l'occasione per discutere della lingua volgare. Il Tolomei esordisce lodando la lingua toscana, che non ha bisogno di ornamenti provenienti dal latino o dal greco, poiché «assai bella è per sé et assai ornata de' drappi suoi»; al latino e al greco devono invece rifarsi gli altri volgari della Penisola, «se vogliono parer madonne e non contadine»(p.19). Lo contraddice il Trissino, che biasima l'allontanamento del toscano contemporaneo dal latino e condanna sia la tendenza a introdurre nuovi vocaboli, sia la moda arcaizzante che induce molti a preferire parole antiche e rare.

Dopo aver rilevato la distanza tra il toscano parlato e la lingua scritta usata dai toscani, il vicentino pone un quesito al Pazzi e al Tolomei: la lingua toscana è una sola e si può identificare con il fiorentino, oppure si devono distinguere più lingue toscane, includendo il senese e gli altri volgari della regione nella categoria più generica di «lingua toscana»? Il Pazzi riconosce che fiorentino e senese si differenziano poco, più negli aspetti fonetici che nel lessico; il Tolomei «punta invece l'attenzione sulla distinzione diastratica, che percorre tanto il fiorentino quanto il senese ${ }^{25}$, cioè ritiene che il volgare di un parlante senese colto non sia inferiore a quello di un fiorentino di pari condizione.

21 La discussione scivola a questo punto sull'origine del volgare, secondo il Trissino derivato dal latino, che è, dopo il greco, la lingua «più elegante, più culta, più castigata, più copiosa» (p. 24). La lingua sviluppatasi dal latino, comune a tutta l'Italia, ha poi subito l'influsso del greco bizantino, al quale si devono l'introduzione dell'articolo e il doppio timbro delle vocali e e o. Gli uomini di giudizio, consapevoli che nessuno dei volgari è in sé perfetto, usano quello che più di ogni altro si avvicina al latino, cioè la lingua cortigiana. Il toscano, invece, viene spesso considerato adatto soltanto ai canti carnascialeschi e alle commedie. In polemica con il Tolomei, il Trissino afferma quindi 
che il volgare migliore è quello che più s'accosta alla lingua madre; singolare è a tal proposito il giudizio espresso sullo spagnolo, considerato un latino invecchiato, una degenerazione del latino, a differenza dell'italiano che ha con esso un rapporto di filiazione («la nostra volgare è figliuola e procede dalla sostanza medesma della latina, benché alquanto diversa di forma», p. 27).

Segue il lungo intervento del Tolomei sulla questione dell'origine del toscano, che deriverebbe dall'etrusco: «Et come questa vostra commune italica dite esser derivata dalla latina, così la toscana moderna potemo creder che venga dall'antica lingua etrusca, la qual hoggidì è spenta affato, forse per la violenza della nuova $o$, com'è più credibile, per la fortuna del grande Imperio Romano» (p. 29). Questa opinione non è condivisa dal Tebaldeo; il Pazzi, infine, pur aderendo alla teoria dell'origine latina, riconosce il debito che i Romani hanno contratto con gli Etruschi.

Prende quindi la parola il letterato ferrarese, affermando che nessuno scrittore toscano degno di rilievo ha scritto nella lingua che viene parlata in Toscana. Alla reazione del Pazzi, che cita Dante, Petrarca e Boccaccio, risponde ricordando che Dante «abbracciò tutte le lingue d'Italia, in che consiste questo nostro idioma italico» (p. 34) e Petrarca, pur essendo nato ad Arezzo, ebbe un contatto assai limitato con la Toscana; indubbiamente il Boccaccio scrisse la sua raccolta di novelle in fiorentino, volgare che si addice, appunto, ad argomenti comici e a storie destinate a un pubblico femminile. La posizione cui il Valeriano dedica maggiore spazio e rilievo è quella del Trissino, il quale, per spiegare il carattere composito della lingua italiana e il rapporto che intercorre tra essa e i volgari regionali, si serve del paragone con il corpo umano (si veda il secondo estratto). Quanto alla lingua del Petrarca, gli argomenti su cui fa leva il Trissino personaggio coincidono con quelli esposti dal Trissino autore del Castellano: il grande trecentista ha usato la lingua comune, adottando solo poche parole toscane, facilmente riconoscibili perché «le parole che sono più corrotte o più stroppiate, quelle sono le toscane» (p. 43). A proposito del nome da attribuire alla lingua, poco oltre impiega gli aggettivi cortigiana, italica e commune come se fossero intercambiabili.

In che modo dovrà quindi regolarsi il letterato colto e d'ingegno? Dovrà attingere al lessico di tutti i volgari italiani, selezionando i vocaboli migliori, e seguire l'esempio dei valent'homini e dei buoni libri. All'obiezione del Pazzi che la lingua toscana possiede un vocabolario così ricco da poter ricoprire tutti gli ambiti della comunicazione scritta, il Trissino risponde che si possono definire toscane solo le parole che si usano esclusivamente in Toscana, non quelle presenti anche nelle altre regioni, che sono invece proprie della lingua comune. Vocaboli come corpo, regno, versi e fiumi, per esempio, non sono solo toscani, ma anche lombardi, romani e di altri luoghi d'Italia.

Il Pazzi, a questo punto, gli chiede un chiarimento in merito alla presunta discrepanza tra la lingua parlata dai fiorentini e il volgare letterario, dubitando che vi possano essere delle differenze. Il Trissino affronta la questione sottoponendo l'amico fiorentino ad una prova: lo invita a leggere a voce alta alcuni versi di un sonetto petrarchesco che contengono la parola laudando. La pronuncia del Pazzi (laldando) in effetti non corrisponde alla scrittura e ciò dimostra - secondo il letterato vicentino che la lingua parlata a Firenze non coincide con il volgare letterario del Canzoniere. Il Trissino può quindi concludere che «Il Petrarca adunque non ha detto laudando né laudare come toscano, ma come lingua commune accostandosi al latino» (pp. 48-49). La lettura di un altro verso petrarchesco, che contiene la parola gloriosa - groliosa nella pronuncia del Pazzi - conduce ad un'ulteriore conferma dell'assunto trissiniano ${ }^{26}$. La 
mancata coincidenza tra toscano scritto e parlato è del resto un argomento affrontato a più riprese nel Dialogo, come sottolinea Claudio Giovanardi ${ }^{27}$.

Concluso dai versi finali della canzone CCCLX di Petrarca («Piacemi aver vostre quistion udite, / ma più tempo bisogna a tanta lite», vv. 156-57), pronunciati dal cardinale per congedare gli ospiti, il Dialogo resta aperto, anche se l'ultima parola spetta al Trissino ${ }^{28}$. Egli introduce la distinzione tra il fiorentino popolare e la lingua colta usata dallo stesso Alessandro de' Pazzi, da Luigi Alamanni, da Bartolomeo Cavalcanti e da «alquanti altri vostri pari giudiciosi e veri imitatori del Petrarca e del terso et elegante parlare», che «tess[ono] di trama italica e cortegiana» e dal volgare nativo traggono solo «certe fila per farne fregi e ricami, e questo anche con gran modestia e discretione», differenziandosi dagli intellettuali alla moda che toscaneggiano in modo affettato (p. 51). L'ultimo intervento del vicentino si chiude quindi con una ripresa della critica avanzata dal Marostica nella cornice.

\section{Pierio Valeriano, Dialogo della volgar lingua}

\section{Criteri di edizione}

\section{Fonte}

Viene seguito il testo della princeps: Dialogo della Volgar Lingua. Di Gio. Pierio Valeriano bellunese, non prima uscito in luce. All'illustr.mo et Reverend.mo Sig. il Principe Don Carlo Cardinal de' Medici. Con licenza de' Superiori, et Privilegio, in Venetia, nella stamperia di Gio. Battista Ciotti, $1620^{29}$.

\section{Criteri di trascrizione}

Criteri di trascrizione: si sono conservati i caratteri grafici originari del testo, l'h etimologica, il grafema $j$-riscontrabile come secondo elemento nella coppia ii in posizione finale (prodigij) - e le divisioni delle parole, ad eccezione di de $i>$ dei e di poiche (con valore temporale) > poi che. È stato uniformato alla norma grafica moderna l'uso delle lettere $v$ e $u$. Le abbreviazioni dei nomi e di messer sono state sciolte e si è impiegato il corsivo per le forme linguistiche citate nel testo. Gli accenti, gli apostrofi e le maiuscole seguono le norme grafiche moderne. Gli interventi sull'interpunzione, infine, sono stati modesti e funzionali alla comprensione.

Il testo a stampa del 1620 è numerato per pagina. Il numero di pagina viene messo tra parentesi quadra; se la parola è spezzata tra una pagina e l'altra, la numerazione viene inserita prima del cambio di pagina.

\section{Testo integrale: Pierio Valeriano, Dialogo della volgar lingua}

\section{All'Illustrissimo et reverendiss. Signore il Principe Don Carlo Cardinal de' Medici}

31 [3] Gli scritti del Pierio sono dediti alla Casa Serenissima de' Medici per l'educatione e coltura dello 'ngegno ch'egli hebbe in essa prima appresso il gran Lorenzo in una ragunanza di tanti huomini di lettere, poi seguitando il servitio e la fortuna di Leon Decimo e di Clemente Settimo fin all'ultima sua età. Ma fra l'opre di tanto Scrittore niuna sen'è trovata in volgar lingua, fuorché questo Dialogo che di essa apunto ragiona. 
E perché tra le fortune di questa vita io [4] stimo singolarmente l'haver havuto la patria commune col Pierio e commune, se non la familiarità della Casa de' Medici, almeno l'honor d'esser vivuto in essa alcun tempo, che vi dimorai appresso il Signor Paolgiordano Orfino; ho stimato d'adempier gli oblighi del Pierio e miei in far che questo Dialogo esca alla luce sotto 'l nome di Vostra Signoria Illustrissima; né ho dubitato che sia per ricusar la protettione hereditaria dell'opre del Pierio, né accusar il giudicio et ardir mio in dedicargliele, se ben in questi ragionamenti pare che s'abbassi la lingua toscana. Peroché si riprende sol quella ch'è nell'uso del vulgo, non degli huomini di lettere e di corte; et è costume degli animi grandi l'udir volontieri se v'ha nelle lor cose alcun difetto; e 'l Dialogo ridonda delle qualità del suo autore di 'ngegno, d'arte e d'erudition singolare. Onde mi persuado che Vostra Signoria Illustrissima sarà per leggerlo con quel diletto che l'udì già quel gran Cardinale de' Medici, che poi fu degno per le sue virtù d'esser assunto in tempi difficilissimi al sommo pontificato $\mathrm{e}$ governo di santa Chiesa. Io certo spero dalla degnità dell'autore e dell'opra conseguire che sia grato a Vostra Signoria Illustrissima questo cenno della divotione e servitù mia e, facendole [5] humil riverenza, le prego da Dio ogni corso di felicità.

Di Venetia a' 16 gennaro 1620.

Di V. S. Illustriss. Reverendiss.

Humiliss. et Devotiss. Servitore

Panfilo Persico.

\section{A' LETTORI}

[6] Questo Dialogo già più di cent'anni scritto è stato raccolto quasi dalle ceneri e reliquie del suo autore per opra di Monsignor Luigi Lollino Vescovo di Belluno, e nella sua copiosissima libraria riposto; dove non già come negli horti dell'Hesperidi i pomi dell'oro, ma a commodo commune in libera custodia si guardano molti rari scritti nelle migliori lingue che s'apprezzino hoggidì; e da questo archivo delle Muse vi sarà lecito, studiosi lettori, aspettar alla giornata maggiori aiuti ai vostri studi.

\section{Persone del dialogo}

[7] ANTONIO MAROSTICA, ANGELO COLOTIO, LELIO

MAROSTICA. Per mia fé, Colotio, vi portaste bene hiersera, prometteste di venir a cena con noi, e non solo non veniste, ma non mandaste manco a scusarvi. Noi v'aspettassimo fino a notte; tanto che le vivande svanivano e messer Mario rinegava la patientia e tutti ci havereste sentito a borbottare non tanto per la cena, che si guastava, quanto perché non havevamo il zuccaro e 'l pepe vostro da condirla.

colotio. Voi havete mille ragioni, ma neanche io ho il torto, perché volsi venire, ma fui contra il voler mio ritenuto da persona che può forzar me e tutti voi.

MAROSTICA. Se questo non è il barigello, io non so chi vi potesse sforzar di questa maniera, che quant'a me so d'esser così libero di me stesso che niuno potria sforzarmi a voler quello ch'io non volessi.

colotio. E voi ancora come savio et accostumato v'accommodate alle volontà d'altri quando vi par il dovere.

MAROSTICA. Sì, ma non isforzo la volontà mia, quando voglio quel che vogliono gli amici.

colotio. Et io volsi hiersera quel che volse il padron mio. 
MAROSTICA. V'intendo, questo fu un atto della volontà, che vi fece disvoler quel che volevate prima.

colotio. Voi l'havete detto, ma udite come. Io veniva dalla mia vigna, per trovarmi a cena con voi secondo la [8] promessa, e fuori della porta m'incontrai nel Cardinal de' Medici, che andava alla sua vigna con una compagnia d'huomini letterati; et havendogli io fatto riverenza e lasciatol passare, mi messi per venir di lungo, ma sua Signoria mandò un palafreniero a invitarmi, ch'io andassi a cena con quegli huomini da bene. Così colto all'improvisa non seppi e non ardi' ricusar il favore, massime che sapete come io ho bisogno che sua Signoria raccommandi la cosa mia a Leone; onde mi pareva haver havuto buon incontro di poterli così parlar a modo mio. Restava ch'io mandassi a scusarmi con voi e dir che non m'aspettaste, ma non havevo che un sol ragazzo, del qual non poteva far di manco per tener la mula; poi pensai che tra noi non bisognassero cerimonie e che faceste alla libera, essendovi specialmente il Volterrano.

MAROSTICA. Io credo più tosto che vi perdeste nella felicità e che non pensaste a noi, né ad altri. Orsù, bon pro vi faccia, Colotio; voi sete dei favoriti, che che non è vi vediamo incapellato.

colotio. Purché questo vocabolo non perda le prime sillabe, bono verrà.

MAROSTICA. Ben è, voi havete fatto il debito, ma v'havete perduto una facetissima cena, ché messer Mario s'attaccò col Pietrasanta e vi so dir che sene dissero delle belle.

cоцотіо. Credo, perché so quel che sogliono fare, ma neanche la nostra fu muta, né melanconica, perché vi fu una delle più piacevoli dispute che si facessero mai alle cene di Coritio o del nostro Cursio.

MARostica. Non mene meraviglio, poiché so che il Cardinal de' Medici [9] non è di quei signori che vogliono a tavola e da per tutto far adorar la maestà, ma vuol trar frutto dalla conversatione, specialmente degli homini di lettere, che si trattengono da i grandi e s'accarezzano per haver commodità di poter in ogni luogo, in ogni tempo imparar qualche cosa e così venirsi ogni dì facendo più savi per esser non solamente per beneficio della fortuna ma del saper e della virtù superiori agli altri.

colotio. Voi dite bene, e questo fu sempre il costume della Casa de' Medici.

Marostica. Lo so; ma che disputa fu questa, e di che materia? Perché a tavola si suol ragionar di condimenti, di sapori, di vini e di simil cose che appartengono al gusto.

соцотіо. Niente di questo; ma ben di quella parte di noi ch'è istromento del gusto, se ben in altro officio.

MAROSTICA. Del palato volete dire o della lingua; ma in quale officio?

colotio. Vi dirò, per non vi tener a tedio più lungamente: si ricercò se questa lingua volgare era nostra o d'altri, e se l'era toscana o di che paese, e se si poteva scriver in volgare altramente che con forme toscane. Poi si trattò se per lingua toscana s'intendeva solo la fiorentina, e sopra tutto qual convenisse a un galant'homo.

MARostica. Bellissime quistioni e curiose; ma chi furono i disputanti?

colotio. Messer Giangiorgio Trissino, messer Alessandro de' Pazzi, messer Antonio Thebaldeo e messer Claudio Tolomeo.

[10] MAROSTICA. Valorosi campioni, già m'imagino un abbattimento degno d'esser visto; ma ditemi, li doi Lombardi dovevano esser da una parte e li doi Toscani dall'altra. 

volgarmente scrive, chi vuole scriver bene; e li doi Lombardi che italica era, non toscana, quella che gli antichi volgari scrittori usano e li moderni imitar debbono. Ma fra messer Alessandro e messer Claudio era differenza, ché messer Alessandro voleva che la dipendesse in bona parte dal latino e messer Claudio la voleva alienar del tutto, sì che non solamente la non havesse del latino, ma che non dovesse haver pur uno scrupulo né odor di latinità. Fra 'l Trissino e 'l Thebaldeo discordava, ché, approvando ambidoi che la lingua degli scrittori non era soggetta simplicemente alla toscana et ch'era figliola legitima della lingua latina ma allattata da una balia greca; erano poi differenti in questo: che il Trissino differiva assai alla toscana, ma il Thebaldeo voleva che la fosse la più sciocca di tutte l'altre e che in quella non se dovesse scriver altro che farse, cioè cose da ridere, per esser tutta ridicola; di che messer Alessandro perdeva la patientia e messer Claudio poco men ch'impazziva.

questi ragionamenti per poter finalmente risolvere se ho da parlar con la mia lingua o con quella d'altri, ch'è una compassione il fatto mio, ogni volta che ho da scriver a un amico, star a freneticar, s'io ho da usar la mia [11] lingua o mandar per un'altra al macello. Messer Angelo, non si può più vivere, dapoiché son usciti fuora certi soventi, certi eglino, certi uopi, certi chenti e simili strani galavroni; non posso passeggiar per Parione che vengono questi giovanotti dottarelli, barbette recitanti, e stanno ascoltando quel che ragioniamo insieme, e ci puntano negli accenti, nelle parole e nelle figure del dire, che non sono toscane, senza una compassion al mondo, ridendosi di noi, che, se ben havemo messo la barba bianca negli studi, non sapemo quello che mai non ci sognassemo d'imparare. Non dico già che, poiché havemo un Principe toscano e di tal dottrina, virtù e benignità dotato, non debba ogniuno accommodarse, ingegnarse, affaticarse con tutta l'industria che può di fargli cosa grata. Ma io, povero vecchiarello, come posso hora imparar di nuovo a parlare, che, come vedete, mi incominciano cascar li denti? Certo che m'è venuta qualche volta tentatione di partirmi di Roma per non esser tenuto forse per ribello perché non parlo toscano e mi scappa di quando in quando mi e ti.

colotio. Messer Antonio, la cosa non passa in questo modo. Il Principe non ha fantasia, né pensier, né interesse alcuno in questa materia; è homo universale, dotto come sapete in lettere greche e latine, et essercitato in tutte l'arti che appartengono a un vero e gran signore, e si prende piacere d'ogni essercitio d'ingegno, ma particolarmente di queste dispute et osservationi, perché, havendo la lingua nativa e libera, se [12] ride di questi che la mendicano, ma molto più di quelli che la vogliono restringere e limitar tutto il dì e farla star a regola nelle stinche; sì che non pensate che questo si faccia per adularlo $^{30}$, ché tanto amerà egli una cosa ben detta nella cappella di Bergamo, quant'un'altra detta sotto la cuppola di Fiorenza. La quistion è fra questi begli ingegni e scientiati de' nostri tempi.

58 MAROSTICA. Oh, voi mi levate d'un gran pensiero; ma si contenteran ${ }^{31}$ poi questi scientiati ch'io usi la lingua mia nei miei bisogni, o pur vorranno ch'io la cambi con altra a modo loro?

59 CоLотіо. Mi fate ridere; sapete ben che altro ci vuole a viver che lingua. La lingua si dà per antipasto; poi, come dice Martiale, carne opus est, si satur esse velis.

60 MARostica. Voi dite il vero, ma sapete ben anche quel che si dice, che la lingua è la migliore e la peggior pezza di carne che si trovi; però bisogna farne caso, e forsi per questo al paese mio le mettono in sale, e si può creder che sia fatto con misterio, perché 
il sale è simbolo della sapientia, nella quale bisogna che la lingua si purghi e si condisca per preservarsi dalla corruttione et esser più grata al gusto.

$$
\text { lui. }
$$



cominciò dimandar questo e quello delli convittati quello che studiavamo al presente o componevamo. Messer Alessandro disse che rivedeva la sua tragedia; misser Giangiorgio, che stava sopra una fantasia di certe lettere che mancavano nel nostro alfabeto, poiché, havendo la pronuntia diversa, si notavano con la medesma figura. Sapete, messer Antonio, quelle che sono appresso i Greci distinte, che già molte volte n'havemo ragionato insieme e concluso che in effetto è stato gran mancamento degli antecessori nostri non haver osservato una cosa tanto evidente, et haver dato loro la sua forma propria, come fecero i Greci. Piaceva la cosa al Cardinale, e prestava le orecchie molto attentamente; al Pazzi e al Tolomei non entrava questa opinion del Trissino, massime di voler introdur le proprie figure greche, e dicevano ciò non esser necessario, perché la pronuntia s'impara dalla natura e dall'uso cotidiano; e sono molt'altre cose che non si scrivono e si proferiscono, come gli accenti, per essempio parlo in persona prima del presente, come dicono li greci paroxitono, e parlò in terza persona del passato, oxitono, le quali cose s'imparano communemente parlando. Il medesmo esser delle vocali, che i fanciulli imparano a proferir dal padre, dalla madre, dalla nutrice con la differenza che vanno pronuntiate, come erto con la e stretta, certo con la e larga, e così schena e scena, hora, dimora, sola, parola e simili, che l'uso insegna senz'altra distintione a proferir con bocca più chiusa $o$ [17] più aperta, più dolce $o$ più grave: onde la industria e fatica di tal inventione esser inutile e vana.

Il Trissino difendeva l'opinion sua con quest'argomento: che i Greci, i quali diligentissimi furono in ogni cosa, non havevano senza causa fatto tal distintione e, se i Latini non l'havevano accettata, era perché appresso di loro queste vocali si 
proferiscono sotto un singolar sono; ma hora che la latinità ha prodotto una lingua commune a tutta Italia, e li Greci, overo Romani ingrecaniti, che vennero da Costantinopoli a Roma, scacciati i Longobardi, raccolsero questa fanciulla novellamente nata e l'allattarono et allevarono, quindi avviene che ella senta tanto della natura della nutrice. Percioché col latte trasse dalla lingua greca gli articoli, che senz'alcun dubio sono greci, e da Piemontesi e Genovesi si proferiscono più alla greca che da noi, li quali siamo più verso il levante; conciosiaché dicano essi o quello che noi dicemo lo et $a$ quello che noi la. Il che penso haver fatto li nostri per far il parlar più maschio, perché senza le consonanti riesce evidentemente più molle et effeminato. Così dunque, essendo la lingua nostra volgare di genitura latina, di education greca, non è meraviglia se dalla culla imparò due sorti di $e$ et di o, le quali scrivendo noi al modo di quelli che ci hanno dato parte della lingua, non mi par cosa nuova né a sproposito, come alcuni la fanno considerando poco i fondamenti e le ragioni; e tanto più che da quattrocent'anni in qua tal distintion di figure e di sono è restata [18] ferma in tutti i libri greci e nella pronuntia degli homini dotti.

Poi che hebbe così detto il Trissino, messer Claudio volto al Cardinale disse: «Monsignor Reverendissimo, quando non si dia noia a V. S., si contenti concederci licenza che, poiché siamo entrati in ragionamento della volgar lingua e si trovano qui persone di diversa opinione, come altre fiate habbiam conteso, ci sia lecito disputarne con buona grazia sua». Il Cardinal rispose: «Anzi, io non potrei haver maggior piacere che sentir trattar di questa materia; e quando ne fosse caduto proposito, non haverei messo in campo altro argomento, massime ritrovandosi qui tali huomini e tanto essercitati in simili quistioni. Dite dunque, ch'io ven'haverò particolar obligo».

Quivi il Thebaldeo, che sapeva come messer Claudio era per dir gran cose in essaltation della lingua toscana e che soleva anteporla alla latina, così parlò: «Monsignor Reverendissimo, e se nel ragionamento, mentre si cercherà la verità, accadesse d'abbassar la lingua toscana e posporla a molt'altre, V. S. Reverendissima si degnerà di perdonarci?». Rispose il Cardinale: «V'ho detto che non solo vi perdono, ma ho caro e vi prego che ogniun dica il suo parere; et non habbiate rispetto ch'io sia nato in Firenze; anzi, se cosa è che non accettiate delle nostre, ditela pur liberamente, perché, errando forse noi, ci emendaremo. Io non fui mai tant'ostinato in difender una ragione che la volessi vinta a dritto o torto; et vi dirò che 'l parlar di molti nostri [19] Fiorentini spesso mi fa ridere, ma, quando sento un cortigiano dotto et di giudicio, mi piace assai più ch'e nostri et vi riconosco una certa gratia, la qual non vi saprei dir, ma sol la sento. Vero ha ch'e nostri, i quali si sono allontanati dalla Cuppola et hanno pratticato fuori, mi paiono poi mirabili. Sì che in questa materia mi trovarete neutrale».

Replicò il Thebaldeo: «Questo dico, Monsignor Reverendissimo, perché messer Claudio, per quel che molte volte havemo contrastato insieme, vuol che la lingua volgar debba, quanto può, discostarse dalla latina, e che la figliuola di così bella madre non habbia alcuna somiglianza con lei; il che, oltre che dà sospetto di suppositione, pare pur ancor cosa contra natura».

Soggiunse allhora messer Claudio: «Io il dico perché non ha la lingua toscana bisogno dell'altrui bellezze, né d'ornamenti forastieri; ché assai bella è per sé et assai ornata de' drappi suoi. Accostinsi pur alla greca e alla latina la genovese, la lombarda, la romagnola, la marchiana, la pugliese, la calavrese e parte della Terra di Lavoro, e la romanesca ancora, perché n'hanno bisogno, se vogliono parer madonne e non contadine». 
Quivi il Trissino: «Buone parole per vita vostra, messer Claudio, ché ce n’è anche per voi. Non so come voi cominciate il giudicio innanzi la citatione e vi costituite in possessorio senza veder per chi è la ragione; non credo che i vostri iurisconsulti v'habbiano insegnato tal modo di procedere».

Messer Claudio rispose: «Io parlo così perché sono in tal materia risolutissimo» [20].

TRISSINO. Non è possibile che una bella cosa si possa far più bella? È mai l'oro tanto fino che non si possa affinare ancor più?

TOLOMEI. Quando l'oro è alla lega del fiorino o ducato ongaro o venetiano, è venuto dove può, né vi si ricerca più finezza; così la lingua nostra non pote migliorare per opra d'altri più che per se stessa.

TRISSINO. E pure i vostri auttori non solo si servono della lingua latina ma della lombarda, della romagnola, della marchiana e d'altre.

TOLOMEI. Niente, tutto è suo e nato in casa sua.

TRISSINO. Hor volete ch'io vi dica, messer Claudio, tanto havete fatto voi et alcuni altri da un tempo in qua, che havete guasto la vostra lingua e, mentre che andate trattando e ritrattando, l'havete imbastardita e d'una venerabile matrona fattala diventar una bagascia. Li vostri antichi scrittori hebbero giudicio e conobbero la castità della latina, e presero cura che la figliuola s'assomigliasse alla madre quanto più si potea; voi, con questo vostro discostarvi dalla latina, stroppiate le voci e i modi di scrivere e, fatta tra di voi una nuova lingua quasi in forma di gergo, la battezate per toscana, e non è così.

TOLOMEI. Oh! Poniamo che sia come dite voi: s'è lingua composta da Toscani et usasi in Toscana, come non è ella toscana? Che opinione è cotesta vostra? Non mi meraviglio se l'altrieri in Belvedere dicevate ancora che 'l Petrarca non ha scritto in lingua toscana.

TRISSINo. Del Petrarca ch'io dica il vero, vederemo poi; della [21] lingua toscana dico che tanto peccate voi con la novità delle parole e vocaboli strani ch'introducete, quanto peccano quelli che van cercando detti troppo antichi e pongono l'eccellenza del dire in voci oscure e non usate; sì come avvien nel latino, che molti pecchino per troppa licenza, altri per troppa stittichezza d'obligarsi all'antichità e seguir cose e parole astruse e non intese. Sì che concludo che, poiché dite la lingua toscana esser la bellissima, essendo quella che voi hora usate non molto bella, però non è toscana; overo perché si dilunga dai buoni auttori toscani, non è toscana; overo perché voi toscani non scrivete come parlate, la vostra lingua non è toscana. Penso che hormai m'habbiate inteso.

TOLOMEI. Hor qui non basta pensar così; bisogna, messer Giangiorgio, dimostrar e provar che così sia.

B TRISSINO. S'io debbo provar questo, vorrei prima saper da voi, messer Claudio e messer Alessandro, se sete d'accordo che la lingua toscana sia quella che si chiama volgar fiorentino, nel quale si scrivono ogni dì tanti romanzi e novelle; o se havete tra voi, Fiorentini e Sanesi, differenza nella lingua e nel comporre, come havete nel governo per la emulatione della vicinità. Perché se non convenite in una medesma lingua, sarà forza che siano più lingue toscane: toscana fiorentina, toscana sanese, toscana pistoiese, toscana lucchese, pisana, aretina, perugina, viterbese et altre; e così bisogna ch'io mi pensi diventar un Hercole, se vorrò combatter con tal Hidra. Ma se v'accordate che la vostra lingua sia una sola, e mi date uno schizzo d'essa sopra tale o tale auttore, io mi sforzarò, senz'altra trasformatione, combatter come huomo e, benché debole di 
piedi, spero che correrò ben la mia lancia. Però prima che passiamo più inanzi, risolvetemi fra voi se la lingua sanese è la medesima ${ }^{32}$ con la fiorentina, e così dell'altre; o almeno, se quando si dirà fiorentina, s'intenda ancor la sanese; e così, poiché in Toscana non è hoggi altro dominio che importi, essendo voi doi d'accordo, si potrà difinir della lingua toscana senza equivocatione.

PAZZI. E' non è dubio che nella maggior parte convenimo tanto nella lingua, Fiorentini e Sanesi, quanto nell'altre cose siamo differenti; ma questa poca diversità che c'è nel favellare consiste più tosto negli spiriti et accenti che ne' vocaboli.

TOLOMEI. Messer Alessandro, vorrei che distingueste da Sanese a Sanese e da Fiorentino a Fiorentino, perché io non intendo esser discordante da messer Alessandro de' Pazzi nella lingua, né da altri suoi pari. Più presto vi sarà diversa opinione dell'origine, perché io non sento con voi per alcun modo che la nostra lingua habbia del greco né del latino; alla qual persuasione mi pare che troppo facilmente i dì passati vi lasciaste indurre.

PAZzI. Volete voi ch'io nieghi una cosa tanto manifesta?

TOLOMEI. Io non so come manifesta; so bene che né mio padre né mio avo né atavi, abavi e forse quanti ne sono più alti, non seppero mai pur l'alfabeto greco; e si [23] visse e si scrisse e disputossi delle cose del mondo, della natura, dei costumi, dei governi, delle cerimonie, degli dei, dei prodigij, dei folgori e di mill'altre discipline eccellenti da' Toscani senza lettere greche. E Dardano, figliol di Giove nato in Toscana, passò in Candia, e quivi e nel resto della Grecia sparse nostre lettere, nostri istituti, nostre leggi, nostre discipline, le quali poi i Greci usurparono per sue, tacendo invidiosamente gli auttori da chi havute le havevano; quel che non fecero i Romani.

PAZzI. Li Romani non potero nasconder quello che riceverono da' Toscani, perché dal bel principio li Toscani furono fatti Romani, e la somma potestà et honor fu con essi communicata, e fu poi sempre una medesma cosa il Latio con la Toscana. Ma non siamo noi adesso su questa repetitione d'antichità; siamo sopra la lingua d'hoggidì, la qual hebbe principio da seicent'anni in qua, e forsi molto meno.

тоLомеI. Se volemo ricercar questa cosa tanto minutamente, non solo ci bisognerà dir che una terra di Toscana sia differente dall'altra, ma ogni quartiero, ogni famiglia, ogni casa, e nella medesma casa altro esser il parlar del padre da quel dei figli, del marito dalla moglie e dei medesmi fratelli fra di sé, altro nella medesma persona da un'età all'altra, da uno stato all'altro, non essendo cosa più propria alla natura delle cose del mondo che la varietà; di maniera che o la voce o li gesti o li modi di dir o gli accenti, che ancor senz'avedercene ci s'attaccano spesso dal pratticar [24] con diverse persone, sempre inducono nel parlar nostro qualche novità. Onde il voler badar ad ogni minutezza in simil quistione non saria altro che un procedere in infinito.

PAzzi. Adunque bisogna dar una certa forma di parlar in che consista la lingua toscana.

TOLOMEI. Ella mi pare tant'ampia e spatiosa ch'io non so come possa chiudersi in angusto spatio di circoscrittione.

PAzzi. Chi dicesse così, che la lingua toscana è idea della latina, con modi atti alli tempi nostri accommodata?

108 TOLOMEI. Non consentirò in alcun modo che si ponga ch'ella derivi dal latino, né habbia da far con Roma, se non quanto la Toscana le mostrò di ragionar e sapere.

PAZzI. Se volemo arrogarci questo, ci concitaremo adosso grand'invidia. 
110 TOLOMEI. Or fate voi; io per me non son per mettervi la fava nera, né impacciarmene più; fate pur a modo vostro. TRISSINo. Saria tempo, messer Alessandro, d'haver tratto messer Claudio d'errore. Voi confessate pure che la nostra volgar lingua sia tratta dalla latina e che dopo la greca non si trovi la più elegante, più culta, più castigata, più copiosa della latina? PAzzi. Senza indugio alcuno lo confesso e senza difficoltà, se non è che gli Ateniesi sono riputati barbari dagli Scithi.

113 TRISSINO. Hor fra le cose belle, poniam per essempio fra dieci o dodici statue di Mercurio o di Pallade fatte da diversi artefici che ve ne sia una bellissima, non direte [25] voi che quella che più si rassomiglia alla bellissima sia più bella delle altre inferiori?

114 PAZzi. Senza dubio.

115 TRISSINO. E nel medesmo modo direste più brutta quella che alla bellissima è più dissomigliante? PAZzI. Questo ancora è manifesto. TRISSINO. Voi che sete cortigiano et havete pratticato diversi homini letterati, havete mai udito lingua che più s'accosti alla latina di quel che fa la cortigiana in bocca d'homini giudiciosi e non affettati?

118 PAZZI. In vero nella corte di Ferrara, d'Urbino e di Roma ho trovato gli homini letterati parlar in questo volgar, se non toscanamente, certo con grata, dolce et acconcia maniera di dire, et esprimere i concetti suoi con ogni soavità.

119 TRISSINO. E quando li vostri parlano in simili conversationi de' letterati, se non sono di quelli che hanno pratticato fuori lungo tempo, sentiste mai la più inetta e spiacevol cosa che udirli?

120 PAZZi. Certo, io v'ho pensato più volte e men'arrossisco in servitio loro, e per me faccio ogni cosa per non mi lasciar portar dal naturale a quell'uso del parlar che appresi da fanciullo.

121 TRISSINO. Questo nasce in voi e negli altri homini di lettere, perché, conoscendo niuna lingua esser perfetta in se stessa e volendone far una laudevole, vi servite della latina, accostandovi quant'è possibile senza passar i termini della volgarità. Et li Toscani, che vogliono discostarsene nei vocaboli, nella pronuntia e negli accenti, riescono ridicoli et inetti; né meraviglia è se la lor [26] lingua non si mette in publico se non in persona di hosti e di vignaroli e di ragattieri, ogni volta che di carnovale si fanno le mascare e le comedie; perché il commune tiene ch'ella sia lingua da persone di simil affare. Onde voi altri letterati ven'allontanate, e messer Claudio medesmo, che si mostra tanto ribello della lingua latina, è più ribello assai della toscana; perché di quanti ho fin al giorno d'hoggi pratticati, niuno ha più scelte le parole, più puri e spiccati gli accenti, più soave la voce, più accommodato il moto, i gesti, e più gratiosa e convenevole l'attione; e sa pur egli che non trova queste sue doti se non apena in tre o quattro altri Sanesi o pari o imitatori suoi.

122 TOLOMEI. Vi ringratio, messer Giangiorgio, della buona opinione che mostrate haver di me, e la voglio riconoscer più tosto dalla humanità e cortesia vostra che imputarla a malizia che habbiate di tirarmi per questa via nel vostro senso. Ma per risponder a quel che mi cuoce, non vedo, né riconosco nel ragionar mio cosa che non sia toscana, e non so come voi presupponete saper di me più di quel che veda io medesmo. 
TRISSINO. Non è persona che in giudicar di se stessa non possa errare; sempre la propria affettione offusca la ragione, et ogniuno ha qualche particolar discorso che non è commune con intelletto d'altri. Per questo è prohibito al medico, per savio e valente che sia, di medicar se stesso, perché se ha per concluso che ogniun s'inganni nelle cose proprie; e i vostri leggisti ancora commandano che in causa propria l'huom deva trovarsi un avvocato [27].

TOLOMEI. Vedete, messer Giangiorgio, che voi mettete in campo una quistione, che trahe in pericolo non solo la nostra, ma ancora la vostra lingua, che voi difendete, e così averrà che fra dua litiganti un terzo sene porti il pregio.

TRISSINO. Qui non v'entra terzo, perché ponendo la lingua latina per la prima, la seconda sarà quella che più le s'approssima.

тоLомеI. Adunque la perderemo ambidue insieme, perché, se vi si concede che sia più bella la lingua che più s'approssima alla latina, siamo spediti noi e tutte le lingue d'Italia insieme con la vostra cortigiana, perché la spagnola senza dubio otterrà il principato, la qual non mi negarete che non solamente riserva infiniti vocaboli della latina, ma ancor buona parte delle declinationi.

TRISSINo. Noi non dovemo dir che la lingua spagnola s'approssima alla latina, ma ch'è propria latina, ma invecchiata e tanto per l'età mancata che ha perso i denti e la vista $\mathrm{e}$ l'udito; e perciò manca dei sentimenti e non proferisce ben le voci.

TOLOMEI. Il medesmo diremo della volgare.

TRISSINO. Gran differenza è, messer Claudio, a dire esser similitudine da un figliuolo al padre dal dire esser similitudine da Socrate giovane e prosperoso a Socrate già invecchiato; poiché, come sapete, altro è l'esser diverso, altro l'esser diversificato. Così dico io che la lingua spagnola è la medesma latina, come quella che in tante colonie romane v'è rimasta. Ma la nostra volgare è figliuola e procede dalla sostanza medesma della latina, benché alquanto diversa di forma.

TOLOMEI. Come? Non è corrotta anche la spagnola?

TRISSINO. Secondo le persone che parlano, accade alla [28] spagnola come all'altre lingue, che sono tutte corrotte in bocca del volgo; ma dove sia dottrina, ingegno e diligenza tutte s'acconciano in buona forma; così chi volesse metter cura nello scrivere e parlare spagnolo, scriverebbe e parlarebbe simplice latino; et a' Romani che spagnolo non sapessero pareria latino, a' Spagnoli che non sapessero latino pareria lingua loro volgare.

Replicò messer Claudio: «E come si potria far questo?». «Facilmente» rispose il Trissino «e questa materia al tempo del Duca Valentino in Roma fu disputata e messa in esperienza così in prosa come in rima; e di molte cose ch'io viddi allhora mi ricorda ancora un principio d'una lunga ballata, la quale vi recitarò per giustificatione di quanto ho detto. Ascoltate, se non vi pare d'udir qualche hinno di Numa Pompilio:

Contrarias gratias amando,

contra amores procurando,

tu me condennas amor

de falsa gloria dolor,

falsas causas allegando.

o condennationes tales,

o passiones infernales,

de tan continuo tormento

de momento a momento 
das amor penas mortales

malamente me trattando,

de potentia condennando

me matas, o false amor» [29]. TOLOMEI. Non vi concedo, messer Giangiorgio, che la lingua toscana sia peggior della cortigiana $\mathrm{o}$, come voi dite, della commune, perché si discosti più dalla latina; né vi concedo che la toscana venga dal latino, perché è lingua propria e separata e independente et ha le sue proprie inflessioni e forme e figure et eleganze di dire forse assai più che non ha la latina. Et come questa vostra commune italica dite esser derivata dalla latina, così la toscana moderna potemo creder che venga dall'antica lingua etrusca, la qual hoggidì è spenta affato, forse per la violenza della nuova o, com’è più credibile, per la fortuna del grande Imperio Romano, il quale ogni cosa trasse in se stesso et [30] assorbì, come l'oceano assorbe tutte l'acque, perché volevano i Romani, come ben sapete, che tutte le cose publiche latinamente si trattassero. Ma che direte voi, messer Giangiorgio, s'io vi dimostrarò che la latina ancora de' boni tempi fu toscana? Non sapete voi che i Romani antichi non havevano lettere né discipline, se non quelle che i lor figli, che eletti dal Senato si mandavano in Toscana per imparare, a casa riportavano? Non dicono i grammatici antichi che Ennio, Lucilio, Pacuvio et altri poeti di quel tempo proferivano le parole che terminano in bus e in mus senza la lettera finale del sibilo con pronuntia toscana? Il che si vede in tanti lor versi che restano tuttavia, benché l'ignoranza de' nostri grammatici ve l'aggiunga. Però dice Capro che li Toscani a pena havevano la $s$ per lettera et la passavano senza suono, come il più fanno li Francesi a' tempi nostri. Onde li Romani, che da' Toscani havevano imparato, molte parole hanno declinato al modo loro in molti nomi della terza declinacione, rifiutando la $s$ finale nel caso genitivo: «bellum Persi Macedonicum», dice Sallustio, non Persis; «Arconidi filiam», non Arconidis, dice Terentio; «in Timarchidi potestate», non Timarchidis, disse Cicerone; e pure nel dativo disse: «neque in praesenti Timarchidi quid responderet habuit»; e 'l medesmo disse «filius Verri», non Verris. Carisis, grammatico antico, riferisce d'haver osservato appresso Plinio nel libro ch'egli scrisse De sermone dubio, «Herculi» in vece di Herculis. Sì che vedete la lingua toscana essere stata molto inanzi [31] la latina et haversi conservato lungamente doppo, anzi essere stata usata per eleganza e per bellezza; e che, crescendo sempre di culto e d'ornamento, è venuta a tale che hora trahe a sé più che mai non solo i vicini, ma tutta l'Italia ad imitarla. Perciò non dite più ch'ella sia corrotta, perch'è sanissima; non dite ch'ella sia invecchiata, 
perch'è nel più bel fiore e nella maggior robustezza che fosse mai. E se voi altri Galli o Lombardi, li quali non havevate se non quel poco di latino, che vi portavano li coloni romani discepoli nostri, privi del corpo, vi sete actaccati all'ossa, e con quelli vi sostentate, doletevi della sorte vostra che altro non vi sia toccato et aiutatevi col latino o col greco a mostrar l'ingegno. Ma non invidiate a noi Toscani i beni e la gloria nostra, affaticandoci invano di sterpar così ferma pianta, la qual ha nello spatio già di sei mill'anni e più mandate le radici fin al centro della terra e li rami fino alla nona sfera del cielo.

Allhora il Thebaldeo, non potendo haver più patientia, voltatosi al Trissino, li chiese di poter risponder quattro parole, e sorridendo disse: «Certamente molto grandi, magnifiche et eccellenti sono le cose che messer Claudio per virtù dell'ingegno $\mathrm{e}$ facondia sua ha alla toscana lingua attribuite. Ma mi ha fatto ricordar la illustre genealogia del Despota della Morea, quando in Campidoglio, dove si recitava il Penulo di Plauto, voleva preceder al Sig. Alberto Pio Sig. di Carpi e Vicario general della Maestà Cesarea in tutta Italia. Quanti nobili signori, quanti principi, quanti titoli allegava detto Sign. Despota in suo favore e del suo sangue. Ma il Sig. Alberto gli rispose che [32] veramente riconosceva quella nobiltà, quella grandezza in quei principi e signori che 'l Despota raccontava, ma che di presente altro non vedeva di quella progenie, se non un povero fuoruscito, che mendicava il vivere, né di tante cose s'era riservato altro che l'honorato nome con una bella barba e un lungo cappello in forma di quel vaso che noi usamo in uso sordido. Così diremo noi della lingua toscana esser quella stata antica, nobile, eccellente, maestra d'ogni virtù, d'ogni dottrina, d'ogni disciplina, d'ogni bon rito e d'ogni bon costume; ma al presente non esserne restato se non il nome, la lingua esser persa, i libri mancati, le discipline andate in oblivione; restar ben qualche ricordanza delle lor lettere, ma non esser persona al mondo che ne sappia legger pur un carattere, cosa che né alle greche né alle hebraiche né alle caldee né all'egittie è intravenuta. Ma s'è creduto messer Claudio ingannarci sotto l'equivocatione, facendo l'argomento: Scipione prese Numantia, Scipione fece Cartagine tributaria, Scipione debellò l'Asia, adunque Scipione, medico nostro romano, è il più trionfal homo del mondo. Onde come nelli Scipioni, distinguendo i tempi e separando le persone, si troverà altro essere stato il Numantino, altro l'Africano et altro l'Asiatico, si vedrà che la gloria loro non appartiene in modo alcuno al nostro medico; così, chi sa qual fosse anticamente la lingua etrusca e le lettere loro e discipline, dirà che non hebbe mai e che non ha che far in conto alcuno con la moderna lingua toscana, [33] della quale noi ragioniamo al presente per determinar come si deve chiamar la lingua nella quale scrivono volgarmente gli homini dotti, e quale havemo noi da imitare $\mathrm{e}^{33}$ farci famigliare. Imperoché qui non havemo bisogno de faul, palatua, falucero, viturgia et altre simil voci dell'antica lingua etrusca, ma d'amore, amicizia, fede, concordia e altri simili che ogni dì vengono in uso. Ma se voleste pur sostener, ch'io non lo credo, che la presente lingua toscana sia quella antica, poca noia mi darà; so ben io questo, che niuno che habbia culto o eleganza, scriverà nella pura lingua toscana. Non farà questo messer Claudio, non lo farà messer Alessandro, non altri che habbiano dottrina e giudicio; $\mathrm{i}$ quali, quando vogliono far qualche ornata compositione, s'allontanano da molti loro modi e vocaboli nativi et accenti et aspirationi, e s'accostano alla commune. Sì che io non so più bello essempio a mostrar che la lingua toscana non è quella che si habbia da imitare scrivendo che veder che niun toscano, di quelli che hanno nome, habbia continuatamente scritto secondo il parlar de' Toscani. E perché dunque dovemo noi obligarci a una lingua che viene dai suoi medesmi rifiutata?». 
Quivi messer Alessandro, riscaldato un poco dalla collera, disse: «Adunque Dante, il Petrarca, il Boccaccio non son homini di nome, di dottrina e di giudicio?».

Rispose il Thebaldeo: «Anzi, perché hebbero nome, dottrina e giudicio non volsero scriver in lingua toscana. Di Dante già constò l'altr'hieri fra noi che, come Homero della Grecia, [34] così egli abbracciò tutte le lingue d'Italia, in che consiste questo nostro idioma italico; onde voi solete accusarlo che rinegasse la patria. Del Petrarca non voleste concedere, né fu tempo di provarvi, che il suo scriver non è toscano; ma sapete ch'egli hebbe poco o niente commercio con Toscana, poiché, nato appresso Arezzo, da ${ }^{34}$ latte fu portato in Provenza, dove s'allevò, imparò et si fece homo; et quivi, havendo raccolti tutti i fiori delle corti et degli scrittori e dicitori eleganti e dotti che lesse e pratticò in diversi tempi, compose quell'ornatissimo festone del suo bellissimo stile. Del Boccaccio, ancorché habbia anch'egli raccolto diversi modi di dir populari d'Italia, nondimeno poco mi curo sel volete o no tutto per voi, perché già havemo detto che non si trova auttor alcuno che habbia scritto in lingua fiorentina, se non frottole, novelle e favole de romanzi, come dicono volgarmente; perché a simili argomenti da far ridere e ragionamenti donneschi ella è molto commoda et appropriata».

lhora messer Alessandro: «Lasciamo star Dante» disse «che non senza causa fu fuoruscito; ma 'l Petrarca che voci o forme di dire ha egli che non siano toscane, ecceto tre o quattro tolte con li medesmi versi da' poeti siciliani, e forse altrettante da' provenzali? Non diciamo noi in Firenze tempo, sospiri, memoria, giorno et altre simil voci? Che manca a lui, dunque, che non ha scritto toscano?».

Rispose messer Antonio: «E queste voci non si dicono anche fuor di Fiorenza? Ma se voi confessate ch'egli habbia imitato [35] i Siciliani e i Provenzali, adunque non s'è obligato alla lingua simplice toscana? Ché quanto all'argomento che fanno i vostri giovani in Fiorenza: il Petrarca fu toscano, adunque ha scritto in lingua toscana, io dico che per questo medesmo argomento seguiterebbe che l'opere di Virgilio fossero in lingua venetiana». Quivi si rise da tutti, e messer Alessandro ripigliò: «Questa è una fantastica propositione; vorrei veder come la difendete».

Rispose il Thebaldeo: «V'ho detto, nel modo che volete provar voi che 'l Petrarca scrisse in lingua toscana. Voi dite: il Petrarca nacque in Toscana, imparò dal padre e dalla madre a parlar toscano, adunque scrisse in toscano. Però se volete voi che 'l vostro argomento vaglia, valerà anche il mio: Virgilio fu venetiano, e doveva in conseguenza parlar venetiano, adunque l'opere sue sono in lingua venetiana».

148 Allhora il Cardinale domandò: «Dove trovate voi, messer Antonio, che Virgilio fosse venetiano, se Venetia fu edificata doppo lui forse cinquecent'anni?».

«Monsignor Reverendissimo» rispose il Thebaldeo «Mantova è una delle più antiche città della provincia di Venetia; et essendo Virgilio nato a Mantova, forza è che fosse venetiano, come il Petrarca, nato ad Arezzo, passa per toscano. Perciò Macrobio, auttor molto stimato, parlando di Virgilio disse: «Unde huius rei Veneto et barbaro poetae notitia?».

Rispose il Cardinale: «Oh, se haveste detto veneto, sarebbe suto tolerabile».

Il Thebaldeo ridendo soggiunse: «Monsignor Reverendissimo, non ho voluto dar [36] questa pugnalata a messer Claudio, al qual pare che si trapassi il cuore, quando nel parlar volgare si dice qualche parola che habbia troppo del latino». Così la cosa si risolse in riso. Ma poi che si furono posati alquanto, il Trissino domandò se poteva rientrar nello steccato, e volto a messer Alessandro cominciò: «Quando si ragiona a 
inquisitione della verità fra homini savi, dotti e modesti, come sete voi e messer Claudio e 'l nostro Thebaldeo, non è cosa tanto difficile che, procedendo per la via della ragione, non se districhi con poca fatica. La via ragionevole è, come voi sapete, per le distintioni e difinitioni; e perciò Platone nostro ci avisa che ogni materia sopra la qual si ragioni prima con certi termini si descriva e segni; però, inanzi che procediamo più oltre, mi par conveniente che si determini che cosa è lingua in genere e che cosa è lingua in specie. E acciò non crediate ch'io vi voglia dipinger questa cosa a modo mio, io voglio star alla difinitione che le darete ambidui voi. Sì che dite, messer Alessandro, se vi piace, che cosa è lingua, della qual hora parliamo».

PAZzI. Mi par che havete fatto bene, e da homo essercitato nelle scienze come sete, $\mathrm{a}^{35}$ metterci per la buona via del disputare. Dico dunque, poiché così vi piace, che lingua è figura di parlare con varie voci, spiriti et accenti, mediante la quale esprimemo quello che nell'intelletto concepimo.

TOLOMEI. Sta bene, ma io la vorrei un poco più breve.

PAZzI. Dite ancor voi il parer vostro [37].

TOLOMEI. Lingua è pittura vocale delle imagini che sono nel nostro intelletto.

TRISSINO. Bene va, sotto diverse parole c'è la medesma sentenza, e fra noi non occorre provar al modo delle scole, se la difinitione ha le sue parti o no, ché tutti la intendemo a un modo, e quanto spetta alla lingua in generale non è qui contrasto alcuno: hor venendo alla specie, che cosa è lingua toscana?

PAZZI. Lingua toscana è forma di ragionar, derivata dal latino, più colta et elegante di tutte le lingue d'hoggidì, la qual ha colto il fiore d'ogni bel detto.

TRISSINO. E voi, messer Claudio, come la intendete?

TOLOMEI. Io la difinisco in questo modo: lingua toscana è fontana viva di dir limpidissimo, non derivata d'alcun'altra, ma sorta in casa sua, la qual ha sparso i suoi rivi per tutta Italia, e della quale quanti valent'homini hanno scritto in volgare si sono tratti la sete, bagnati, imbriacati.

No. O bella metafora, degna dell'ingegno di messer Claudio; volesse Dio che fosse così vera com'è ben detta! Ma è conveniente ch'essaminiamo prima l'opinione di messer Alessandro, poi verremo alla vostra. Ditemi, messer Alessandro, stando la difinition vostra sana e salda, et essendo tanta eleganza nella vostra lingua quanta voi dite, onde aviene che niun toscano scrive con la lingua che parla, o parla come scrive?

PAzzI. Che cosa dite voi?

TRISSINO. Quello che in tutti voi ho notato sempre et osservato; e se pur qualchuno, come certi vostri giovanotti che si fondano solo nella lingua domestica, scrive come si [38] parla nella sua casa, vedete che modi, che sconciature, che spropositi gli escono della penna, e se si può far di manco di ridere.

PAZZI. Oh, questi non entrano nella difinitione, perché non colgono il fiore.

PAzZI. Non intendo che si voglia dir canestri o ghirlande.

TRISSINO. Voglio dir, se voi intendete le semplici parole o la oration colligata.

PAzzi. L'uno e l'altro.

TRISSINO. Con che le ligate voi? 
PAzzI. Con fila delle nostre sete, e 'l più delle volte con fila d'oro.

TRISSINO. D'onde havete tante sete e tant'oro che bastino a tanti lavori?

PAZZI. D'ogni parte del mondo ci vengono queste et altre ricche merci in modo che non mancano.

TRISSINO. Così, pur v'aiutate con merci straniere?

PAzZI. Sì, ma li lavori son nostri e per tutto conosciuti per fiorentini e non hanno punto dello straniero.

TRISSINO. Ma se non fosse l'aiuto della materia forastiera, come fareste voi tanti panni e drappi et altri ricchi lavori?

PAZZI. Certo faressimo meno, ma in somma perfettione.

TRISSINO. Come in somma perfettione? Sono le lane vostre forse migliori delle fiandresche o delle spagnole, e le sete più fine che le bolognesi o le napolitane? Io so che 'l vostro territorio per la maggior parte è arido, e regolarmente le cose che vi nascono devono assimigliarsi [39] alla natura del terreno, in modo che la lana et la seta conviene che siano alquanto asprette ${ }^{36}$.

PAzZI. Anzi è tutto il contrario, ché la tenuità dell'alimento fa che l'humor manco abondi, et così è necessario che li velli si facciano più sottili e più delicati; ma di questo non voglio disputar io, che non son lanaiolo. Benché questa vostra similitudine, messer Giangiorgio, non ha che far col parlare, il quale noi habbiamo abondantissimo, vario e ferace, ché non abonda tanto Trevigi vostro d'acque vive quanto Firenze di parole. Sì che non bisogna far parangone dalla natura del sito alla lingua, ché questa l'habbiamo omnipotentissima.

TRISSINO. Et io vi dico, messer Alessandro, che non si può dar più espressa similitudine al parlare che delli lavori che si fanno in diverse parti del mondo; havemo tutti bisogno di soccorso, e non è idioma alcuno che da sé solo faccia buon'opera, se non s'accompagna con materia forastiera. Voi mi perdonarete ch'adesso sopra di voi medesmo vi dimostrarò la verità, già che non si deve riputar vitio, se in voi manca cosa, il cui mancamento è commune a tutta la patria vostra, dove chi non parla come gli altri è tenuto una bestia, come avien anche altrove. Pigliamo, vi prego, il Petrarca e leggete un sonetto, quale vi pare, ch'io vi farò constar che non leggerete come sta scritto; e se recitarete uno delli vostri, non l'haverete scritto come lo pronuntiate. Dall'altra parte si risponderà a messer Claudio per chiarir che in universale niun toscano scrive nella sua lingua, volendo far opre vendibili [40].

PAzzi. Ecco il Petrarca; poiché son giunto a questo che, doppo tant'anni che ho fatto professione di studioso, s'ha da vedere s'io so legger o no.

TRISSINo. Non si nega, né dubita punto, messer Alessandro, che voi non sappiate leggere; anzi tutti sanno e confessano che per la vostra dottrina e perfettissima cognitione di lettere greche e latine, accompagnate con un pellegrino ingegno e buon giudicio, le opere vostre hanno da insegnar altrui non solo leggere ma scriver ancora. Ma perché sete persona ragionevole, savia e discreta, pensamo che non vorrete per voi né per la patria vostra, se non quel che v'appartiene; quello ch'è d'altri o lo lasciarete alli patroni $\mathrm{o}$, volendovene servire, lo riconoscerete da chi ven'accommoderà.

1 PAzzi. Io non son d'altra opinione, e da vero gentilhomo son per confessar ogni debito che mi sia fatto chiaro doversi altrui. Ma veniamo al Petrarca; ecco il primo sonetto: volete ch'io incominci da questo? 
le membra, e cadauna provincia haver certi vocaboli, certi accenti, certi spiriti, che non convengono con l'altre; ma la carne, il sangue, l'ossa e la pelle esser d'una medesma sustanza in tutte. Chi saprà dunque discernere i denti dall'ongie e gli occhi dalle dita, saprà medesmamente distinguer i vocaboli e le pronuntiationi da provincia a provincia; e quando occorrerà una voce o modo da dir che sia commune, dirà ch'è carne o sangue, e non vorrà che 'l Toscano dica "questo vocabolo è mio perché l'uso anch'io», come se dicesse il braccio «io son vestito di carne, adunque la carne è mia». E così diremo parimente dell'altre provincie e popoli d'Italia. Onde credo havervi fatto vedere come la lingua di Toscana ha ben qualche particolarità ch'è solo sua, ma il corpo del parlar è italico.

TOLOMEI. Io non la intendo in questo modo, perché bisogna distinguer le specie dalla similitudine degli animali, de' quali alcuni nel genere de' quadrupedi sono cavalli, altri orsi, altri elefanti, altri leoni; et a questa somiglianza il popolo toscano chiamaremo leone, perché, com'esso agli animali quadrupedi, così la lingua toscana signoreggia all'altre lingue d'Italia.

TRISSINo. Questa divisione de diverse specie, messer Claudio, si deve fare dove la forma, la natura, la qualità è in tutto diversa, e caderia bene quando distingueste in questo modo la lingua hebraica, la greca, la latina, la todesca, la francese; ma la italiana, se è leone o [43] cavallo, non potrà esser né orso né elefante. Ma poniamo che sia cavallo, non vedete voi quante sorti e forme di cavallo sono al mondo in grandezza, in color, in 
vigorosità differenti? Ma ogni volta che habbiamo li piedi sodi, la coda e le crine lunghe, il capo trigonale, l'hinnito della voce, sarà cavallo e la sustanza sarà conforme in tutti. Il medesmo è del cane, il quale dalla natura è fatto di tante forme e qualità; tuttavia, come abbaia, è cane. Sì che dite più presto la lingua toscana è cavallo di tal o tal razza, di tale o tal sorte, over è cane di tale o tal fatta, che dire che sia di specie dalla italica differente.

PAZZI. Hor sia, in nome di Dio; ma vorrei pure, messer Giangiorgio, che ci mostraste hormai nel Petrarca quello che voi dite, che non ha scritto in lingua toscana; perché havend'io ogni cosa che v'è per toscana, poiché noi usamo tutte quelle forme e vocaboli ch'egli ha usato, non so discerner le parole communi, che voi dite, dalle nostre.

TRISSINO. Facil cosa è mostrarvi questo; aprite il libro e leggete qual sonetto o canzon vi piace, ché non vi trovarete per sonetto o stanza parole toscane da tre o quattro in su; e se pur volete ch'io ve le aditi, vedete le parole che sono più corrotte o più stroppiate, quelle sono le toscane.

192 TOLOMEI. Dirò pur anch'io quattro parole. E' non procede, messer Giangiorgio: è lingua alterata o corrotta, adunque è stroppiata e deforme; perché vedemo tante cose per la corruttione farsi perfettissime, che ben potremo [44] dire che la nostra lingua fosse eccellente e perfetta, ancorché vi si concedesse ch'ella fosse corrotta et in un'altra specie trasformata. Ditemi, per vostra fé, quale stimate voi più perfetto il pane o 'l grano?

TRISSINO. Il pane, senza dubio, perché è il fine per lo qual si prezza il grano.

TOLOMEI. E nondimeno, se 'l grano non si guastasse e convertisse in polvere, poi se ne facesse pasta, e col mezo del lievito non si putrefacesse e se ne formassero le pallote, ultimamente si consolidasse col fuoco, non haveressimo un tal sussidio alla vita nostra. Parimente se quella parte che voi dite nel parlar nostro pigliarsi dal latino non si rompesse, variasse e tramutasse, aggiungendo, minuendo, alterando, non si farebbe così bella lingua chente la nostra. Io ho visto questa sera, fra l'altre delicatissime vivande che Monsignor Reverendissimo ci ha date, esser venuta in tavola una torta, la qual voi, messer Giangiorgio, havete molto lodata e volontieri insieme con tutti noi mangiatone. Ché se tutti quei semplici, che concorrono a far tal vivanda, vi fossero ciascun appartatamente posti inanzi in un piatto, non n'havereste pur assaggiato; ma così guasta e mesticata l'una forma con l'altra v'è sommamente piacciuta. E' bisogna dunque per far una cosa buona sen guastino dell'altre.

TRISSINO. L'essempio che voi ci date, messer Claudio, della torta, fa tutto a nostro proposito per confermation di quello che noi dicemo della lingua cortegiana, o vogliamo chiamarla italica come Dante, o lingua commune al [45] modo de' Greci, come la sua natura richiede; la qual, per far questa vostra torta simil a quella di Monsignor Reverendissimo, piglia le cose da quei luoghi dove sono in maggior perfettione. Perché, come ne insegna Hippocrate, il genio della terra, la qual produce tutti li cibi, non è in ogni luogo il medesmo ma molto vario, come per essempio la Cinerava produce il lasserpicio, che non si trova né in Soria né in Italia. Anzi spesso aviene che una pianta, trasportata un braccio non più da un luogo all'altro, perderà d'odor o di sapore, perché non trova la vena di quel succo del qual è solita nutricarse, ma un altro men proportionato alla sua natura. Colui adunque che sarà spenditor d'un gran signore, volendo le cose in somma perfettione, pigliarà il cascio più tosto da Piacenza che di Sicilia, le spetie più tosto da Venetia che da Pistoia, le frutte più tosto di Fiorenza che di Campagna, le passole di Levante più tosto che da Narni; e così d'ogni luogo si servirà del 
più perfetto che vi si trovi, per far un convito o una cena pontificia et leoniana. Nel medesmo modo il dotto et ingegnoso compositore prenderà il corpo del dire, che sono le parole, di tutta Italia, dove queste o quelle sono più delicate, più significative e più eleganti; piglierà l'ordine della prattichezza de' valent'homini e dai boni libri castigatamente scritti. Da Fiorenza piglierà fioretti, acqua rosata o di fior de melangoli, e qualche bella frutta; e così darà gratia alla sua compositione; in modo che da voi non volemo se non tali cose, o qualche saporetto, ${ }^{37}$ qualche raviolo, ché [46] del resto ne semo, come patroni del tutto, copiosissimi.

Quivi messer Alessandro ripigliò il parlar e disse: «Come? non habbiam noi dovitia di parole da poter servir a tutte le materie che possano imaginarsi per iscrivere, senza questo vostro commune?».

197 TRISSINO. Le havete, sì, ma non sono tutte vostre, perché non havete d'appropriarvi e chiamar parola toscana se non quella che s'usa solo in Toscana, poiché le parole che s'usano parimente negli altri luoghi sono della lingua commune.

PAzzi. Adunque il grano ch'io raccolgo dalla mia possessione a Decimo non è toscano, perché la Puglia fa grano, la Terra di Lavoro fa grano, la Marca, la Lombardia fan grano? Certo questa mi par la più nuova cosa ch'io udissi mai.

TRISSINO. Messer Alessandro, voi che sete bon dialettico, sapete che non procede a dire: Platone ride, adunque tutti quelli che ridono son Platoni; né manco direte voi: il grano di Decimo è toscano, adunque tutti li grani son toscani. Questo è quel che continuamente ho combattuto: che vi volete usurpar e far proprio quel ch'è commune di tutta Italia. Non se vi niega che li beni vostri non sian vostri, e che 'l popolo di Fiorenza, di Siena et altri di Toscana non habbiano e nelle parole proprietà e negli spiriti vivezza e gratia negli accenti; ma se vi dà a credere che corpo, regno, versi, fiumi $\mathrm{e}$ cento migliaia di simili vocaboli sono tanto del lombardo, del romano e d'altre provincie d'Italia quanto vostri [47].

PAZZI. Quali sono adunque quelli che son nostri proprij.

201 TRISSINO. Dirovene alcuni: cinguettare, cavalcione, civanza, tuttatré, tuttaquattro, ambiadua, arrubinargli ${ }^{38}$, melensagine, mentecattagine, impollommi, gratugiati, guagnele, dipocheca, gnaffé, gravaffe e tant'altri simili; e finalmente quelli che non s'usano se non dal vostro popolo o dalla gente di contado. Di questi dite quanto volete che son toscani, ché non vene avemo invidia, perché ogni paese ha le sue proprietà, come nelle cose che produce la natura così nel vivere e nel parlare; benché ad uno che domandasse che cosa è trebiano, meglio si risponderà: «è un delicatissimo vino che nasce in Toscana»; overo che cosa è ravioli: «sono certe formelle di fior di latte che si fanno in Toscana», che a dire «trebiano è vin toscano» o «ravioli sono latticinio toscano».

202 PAzZI. Hor concediamvi quel che volete, benché questa materia si potria meglio essaminare e mostrarvi la soavità di tali parole che a voi paiono tanto istrane, forse perché non le havete in uso; e ritorniamo a quel che mi cuoce, che voi havete detto che non sappiam leggere, overo che in altro modo scrivemo da quel che parlamo; mostratemici un poco questo, che a me è cosa molto nuova.

TRISSINO. Vi si mostrerà; leggete questo.

PAzzi. Ecco lo leggo.

Quand'io movo i sospiri a chiamar voi,

il nome che nel cor mi scrisse Amore, [48] 
laldando s'incomincia udir di fuore

il suon de' primi dolci accenti suoi.

Che cosa è qui che noi altramente pronuntiamo da quello che scrivemo?

TRISSINO. La prima parola del terzo verso, la qual voi proferite laldando et è scritto laudando; e così vuole stare, se dai capiversi di questo sonetto s'ha da raccorre il nome di Laureta. Ma non è sol questo, ché molti altri vocaboli stroppiate nel medesmo modo, né solo fate questo nel volgare, che saria forse tolerabile, essendo volgar vostro, ma lo fate nel latino ancora, proferendo laldes in vece di laudes, galdere in vece di gaudere. E se volete dir che ciò sia per elegantia, tutti di bona voglia ve la lasciamo per vostra sola.

TOLOMEI. Vi si confessa che i Fiorentini non proferiscono laudare né gaudere e simil voci, ma forse quel che a voi par vitio della lingua è fatto per giudicio e per fuggir il sono goffo di quel diftongo au che ha del todesco. Onde dicono più volontieri lodare, lodi, godere, come gli antichi latini dicevano per più gentil sono plostro che plaustro, olla che aula, et hoggidì a questa somiglianza diciamo oro, non auro. $\mathrm{E}$ ben sapete voi che $\mathrm{i}$ Romani amarono tanto questa nostra pronuntia che a un certo gran grammatico, che haveva nome Floro e voleva ritornar in uso questo diftongo con troppa superstitione, $\mathrm{fu}$ beffato da quel gentilhomo, il quale la mattina lo salutò: «Ave, Flaure!».

TRISSINO. Il Petrarca adunque non ha detto laudando né [49] laudare come toscano, ma come lingua commune accostandosi al latino.

ToLomeI. Non mi dà noia il Petrarca, perché la lingua de' poeti non ha regola.

TRISSINo. Come, non ha regola? Se ha scritto, come pur volete, in lingua toscana, non deve obligarsi alle regole di quella, massime dove quella parola non è soggetta a rime, ma stesa per li capi de' versi a suo piacere?

210 TOLOMEI. Noi non habbiamo, né vogliamo haver altre regole, se non di dir tutto quello ci pare ben detto; e ciò ch'è ben detto pensiamo sia tutto nostro.

211 TRISSINo. Che dite voi, messer Alessandro? Non confessate che laudare è latino e non toscano?

212 PAzzI. Io nol posso negare, massime vedendo che i nostri, nol potendo proferir, han voluto dir più presto lodare.

213 TRISSINO. Hor, di gratia, leggete quest'altro sonetto ancora.

214 PAZZI. Leggolo.

Groliosa Colonna in cui s'appoggia.

TRISSINO. Come incominciate?

PAzzi. Groliosa Colonna.

217

TRISSINO. Ma non istà scritto groliosa. Ecco la dolcezza e soavità della vostra lingua toscana, la qual muta la $l$, lettera liquida e soave, nella $r$, aspra e canina; e per più dissonantia trasporta l'aspra per accompagnarla col $g$, lingua corvina non meno sconcia e dissonante. Il che non fate solo in gloriosa ma in molte altre parole; et accioché non iscusiate forse la balbutie o l'abuso della fanciullezza, passiamo più avanti; e se non basta la pronuntia vostra, [50] mirate quella spalliera alla qual s'appoggia Monsignor Reverendissimo, opera antica fino del tempo del Magnifico Cosmo, et in memoria di sì grand'homo riserbata e tenuta cara. Quivi sono tessuti i trionfi delle sette arti liberali, le quali tutte vengono al carro della gloria; e pure sta scritto in lettere molto grandi Grolia. Io ho creduto altre volte che fosse stato error del tessitore in trasportar le lettere fuor del luogo loro; ma, poiché così spesso ho udito et osservato ben la pronuntia 
fiorentina, mi son avisto ch'è di quelle parole che la vostra lingua non cape o non proferisce, e però le guasta.

THEBALDEO. Io non son con voi, che non le possano proferire, ma più tosto giudico che questa sia una razza de vocaboli lor familiari, a lor modo rappezzati, li quali non può proferir se non chi è nato in Fiorenza.

PAzzi. Adunque habbiam pur noi lingua propria e di maggior prerogativa?

TRISSINO. Sì, ma non v'havemo punto d'invidia di quel ch'è proprio vostro.

PAzzi. Perché non conoscete la bellezza della nostra lingua.

TRISSINO. Anzi, perché voi medesmi non la portate in publico, se non purgata col giudicio e dirozata con la lima della corte.

PAZzI. Oh, che cosa possiam noi imparar da' cortigiani?

TRISSINO. Il parlar proprio significante et accommodato alla natura delle cose, pronuntiar le parole che havemo tocco et altre simili. Voi pronuntiate chosa, chasa, con l'aspiratione a gola piena, e come persona dotta [51] scrivete cosa e casa senz'aspiratione, in modo che la corrottione, che messer Claudio vuol rivoltar ad ornato e politezza della lingua, si trova riuscir in contrario, perché li medesimi vostri scritti stanno contra di voi e fan testimonio che vi vergognate di scriver come parlate.

тоLомеi. E' non consiste la lingua toscana in questo parlar popolare, ché ben sapete in ogni luogo esser differente la lingua de' gentilhomini da quella del popolo, e fra li gentilhomini i dotti et esperti parlar meglio che gli ignoranti. Sì che, messer Giangiorgio, perdonatemi, questa mi pare una calumnia voler dissimular tanti bei modi, tante elocutioni ornatissime e colte, et attaccarsi alla feccia del parlar de' lanaioli per abbassar la dignità della illustre lingua toscana.

TRISSINo. Parlando noi della pura lingua fiorentina è pur forza che vi s'intenda il popolar idioma universale, non quel de' dotti come sete voi, messer Alessandro, il Cavalcanti, l'Alamanni et alquanti altri vostri pari giudiciosi e veri imitatori del Petrarca e del terso et elegante parlare, perché tessete di trama italica e cortegiana, e della patria vostra non pigliate altro che certe fila per farne fregi e ricami, e questo anche con gran modestia e discretione. Il che non fanno certi mal aveduti e presontuosi, che si danno ${ }^{39}$ a creder che 'l sceglier vocaboli de' più storpiati che habbiano le pancuzze di Fiorenza, sia la finezza della lingua toscana. Ciò che avenne pochi anni sono anche della lingua latina, ché alcuni si pensarono che 'l suo più bel fiore fosse [52] lo stil apuleiano, forse perch'era manco intelligibile, e così n'empirono tanti scartafacci, finché 'l Pontano, il Sabellico, il Bembo, il Sadoleto et alquanti altri galanthomini comparsero e, con politissimo stil romano cacciati d'Italia questi mostri, rimessero la lingua nella sua natural bellezza e purità.

Quivi Monsignor Reverendissimo, vedendo esser passato un pezzo di notte e che 'l ragionar già passava a qualch'essacerbatione, rizzatosi in piedi disse: «L'hora è tarda et è tempo di ritirarci: Piacemi haver vostre quistion udite, / ma più tempo bisogna a tanta lite». IL FINE 


\section{BIBLIOGRAFIA}

\section{Bibliografia del corpus}

\section{Prima edizione}

Dialogo della volgar lingua. Di Gio. Pierio Valeriano bellunese, in Venetia, nella stamperia di Gio. Battista Ciotti, 1620.

\section{Edizioni successive}

Dialogo del Pierio sopra le lingue volgari: quale sia più conveniente di usare, in Stefano Ticozzi, Storia dei letterati e degli artisti del Dipartimento della Piave, Belluno, Tissi, 1813, pp. 182-217.

La infelicità dei letterati di Pierio Valeriano ed appendice di Cornelio Tollio. Traduzione dal latino. Aggiuntovi altro dialogo originale del Valeriano sulle lingue volgari; ed un capitolo di Cornelio Castaldi contro i petrarchisti, Milano, Tipogr. Malatesta di C. Tinelli e C., 1829.

Il Contarino di Pierio Valeriano e altre opere, Milano, Tipogr. Malatesta di C. Tinelli e C., 1842.

Dialogo della volgar lingua, in Mario Pozzi (a cura di), Discussioni linguistiche del Cinquecento, Torino, UTET, 1988, pp. 39-93 (riproduce l'editio princeps del 1620).

\section{Bibliografia critica}

\section{Studi biografici}

ALPAGO-NOVELlo, Luigi, «Nuove notizie intorno a Pierio Valeriano, con documenti inediti», Archivio storico di Belluno, Feltre e Cadore, VI (1934), pp. 497-504.

BUSTICo, Guido, «Due umanisti veneti: U. Bolzanio e Pierio Valeriano», Civiltà moderna, IV (1932), pp. 83-103 e 344-79.

CAL̀̀, Giuseppe, Della vita e delle opere di Giov. Pierio Valeriano, Catania, Cav. Niccolo Giannotta editore, 1901.

GOUWENS, Kenneth, «L'Umanesimo al tempo di Pierio Valeriano: la cultura locale, la fama, e la Respublica litterarum nella prima metà del Cinquecento», in Paolo PELLEGRINI (a cura di), Bellunesi e feltrini tra Umanesimo e Rinascimento. Filologia, erudizione e biblioteche, Atti del Convegno di Belluno, 4 aprile 2003, Padova, Antenore, 2008, pp. 3-10.

HAIG GAISSER, Julia, Pierio Valeriano on the Ill Fortune of Learned Men: A Renaissance Humanist and His World, Ann Arbor, University of Michigan Press, 1999, pp. 2-23.

LETTERE, Vera, «Dalle Fosse, Giovanni Pietro», in Dizionario Biografico degli Italiani, vol. XXII, Roma, Istituto dell'Enciclopedia Italiana, 1986, pp. 84-88.

LUCCHETTA, Giuliano, «Contributi per la biografia di Pierio Valeriano», Italia Medioevale e Umanistica, IX (1966), pp. 461-76. 
PASTORE STOCCHI, Manlio, «Pierio Valeriano e l'Umanesimo», in Paolo PELLEGRINI (a cura di), Umanisti bellunesi fra Quattro e Cinquecento, Atti del convegno di Belluno, 5 novembre 1999, Firenze, Olschki, 2001, pp. 1-14.

PELLEGRINI, Paolo, «Cenni biografici su Pierio Valeriano», Archivio storico di Belluno, Feltre e Cadore, LXVI (1995), pp. 155-59.

PELLEGRINI, Paolo, «Tra Valeriano e Bembo. Schede d'archivio con tre lettere inedite», Italia Medioevale e Umanistica, XXXVIII (1995), pp. 263-84.

PELLEGRINI, Paolo, «Per la biografia di Pierio Valeriano Bolzanio. Ulteriori contributi», in Lucilla Sandra MAGOGA e Francesco MARIN (a cura di), La certosa di Vedana. Storia, cultura e arte in un ambiente delle Prealpi bellunesi, Firenze, Olschki, 1998, pp. 183-94.

PELLEGRINI, Paolo, Pierio Valeriano e la tipografia del Cinquecento. Nascita, storia e bibliografia delle opere di un umanista, Udine, Forum, 2002.

PELLEGRINI, Paolo e Francesco PIOVAN, «Nuovi contributi per la biografia di Pierio Valeriano», Italia Medioevale e Umanistica, XXXVII (1994), pp. 251-81.

Rozzo, Ugo, «Di Pierio Valeriano e di alcune sue opere», La bibliofilia, CVI (2004), pp. 309-17.

\section{Studi critici}

BONORA, Ettore, «Il "Dialogo della volgare lingua" di Pierio Valeriano e le "Battaglie" di Girolamo Muzio», in Emilio CECCHI e Natalino SAPEGNO (a cura di), Storia della letteratura italiana, Milano, Garzanti, 1974, vol. IV: Il Cinquecento, pp. 182-86.

CASTELLANI POLLIDORI, Ornella, Introduzione a Claudio TOLOMEI, Il Cesano de la lingua toscana, Firenze, Olschki, 1974, pp. 28-31 e Niccolò Machiavelli e il «Dialogo intorno alla nostra lingua», Firenze, Olschki, 1978, pp. 150-51 (per la questione della datazione del Dialogo).

CROCE, Benedetto, «Pierio Valeriano e la controversia sulla lingua», in ID., Poeti e scrittori del pieno e tardo Rinascimento, vol. II, Bari, Laterza, 1958, pp. 74-84.

DIONISOTTI, Carlo, Machiavellerie, Torino, Einaudi, 1980, pp. 300-02 (per la datazione). FLORIANI, Piero, «La "questione della lingua" e il "dialogo" di P. Valeriano», Giornale Storico della Letteratura Italiana, CLV (1978), n. 491, pp. 321-45.

FLORIANI, Piero, «Grammatici e teorici della letteratura volgare», in Girolamo ARNALDI e Manlio PASTORE STOCCHI (a cura di), Storia della cultura veneta, vol. III, pt. II: Dal primo Quattrocento al Concilio di Trento, Vicenza, Neri Pozza, 1980, pp. 139-81.

TROVATO, Paolo, Introduzione e commento, in Niccolò Machiavelli, Discorso intorno alla nostra lingua, Padova, Antenore, 1982, pp. XXXIV-XXXV e LII (per la datazione).

VIANELLO, Valerio, «Le "bellissime questioni e curiose" nel "Dialogo della Volgar Lingua" di Pierio Valeriano», in ID., Il "giardino" delle parole. Itinerari di scrittura e modelli letterari nel dialogo cinquecentesco, Roma, Jouvence, 1993, pp. 87-109.

\section{Studi sulla questione della lingua nel Cinquecento}

CAMPANA, Augusto, «Dal Calmeta al Colocci», in Gabriella BERNARDONI TREZZINI et al. (a cura di), Tra latino e volgare. Per Carlo Dionisotti, Padova, Antenore, 1974, vol. I, pp. 267-315. 
DRUSI, Riccardo, La lingua cortigiana romana. Note su un aspetto della questione cinquecentesca della lingua, Venezia, Il Cardo, 1995 (per il Valeriano si vedano le pp. 27-31).

FORMENTIN, Vittorio, «Dal volgare toscano all'italiano», in Enrico MALATo (dir.), Storia della letteratura italiana, vol. IV: Il primo Cinquecento, Roma, Salerno, 1996, pp. 177-250.

GENSINI, Stefano, «La lingua cortigiana e i dibattiti linguistici del primo Cinquecento», Bollettino di italianistica, n.s., I (2004), fasc. 2, pp. 93-108.

GIOVANARDI, Claudio, La teoria cortigiana e il dibattito linguistico nel primo Cinquecento, Roma, Bulzoni, 1998.

MARASCHIO, Nicoletta, «Il pensiero linguistico nel Cinquecento italiano tra tradizione classica e innovazione», Vox Romanica, LVII (1998), pp. 101-16.

MARAZzINI, Claudio, «Un'idea di lingua: la questione del 'primato' fiorentino», in ID., Il secondo Cinquecento e il Seicento, Bologna, il Mulino, 1993, pp. 149-68.

MARAZZINI, Claudio, Da Dante alla lingua selvaggia, Roma, Carocci, 1999, pp. 37-105.

MENGALDO, Pier Vincenzo, «Appunti su V. Calmeta e la teoria cortigiana», La rassegna della letteratura italiana, LXIV (1960), pp. 446-69.

PISTOLESI, Elena, «Con Dante attraverso il Cinquecento: il De vulgari eloquentia e la questione della lingua», Rinascimento, XL (2000), pp. 269-96.

POzZI, Mario, «Lingua e società: un aspetto delle discussioni linguistiche», in ID., Lingua, cultura, società. Saggi della letteratura italiana del Cinquecento, Alessandria, Edizioni dell'Orso, 1989, pp. 7-27. TROVATO, Paolo, «La questione della lingua e la fissazione della norma», in ID., Il primo Cinquecento, Bologna, il Mulino,1994, pp. 75-121.

VITALE, Maurizio, «La questione della lingua nel sec. XVI», in ID., La questione della lingua, Palermo, Palumbo, 1984, nuova edizione (I ed. 1960), pp. 39-153.

\section{NOTE}

1. Per la vita del Valeriano si rinvia ai testi citati nella bibliografia.

2. Sul Bolzanio si veda il profilo di Lucia Gualdo Rosa, «Dalle Fosse (Bolzanio), Urbano», in Dizionario Biografico degli Italiani, vol. XXXII, Roma, Istituto dell'Enciclopedia Italiana, 1986, pp. 88-92.

3. «Pierio è di casa negli horti del Colocci, sa insinuarsi - apprezzato poeta latino - nella ristretta cerchia del Goritz, la cui villa, visitata da eruditi e antiquari, fu un luogo mitico per la rinascita degli studi sull'antichità pagana, all'epoca di Blosio Palladio. Amico di Pietro Mellini, ha rapporti cordiali persino con i dotti formatisi nell'Accademia romana di Pomponio Leto. Pierio visse dunque tutte le forme di sodalitas culturale poste in atto dall'Urbe nel primo Cinquecento, allora modello per l'Italia colta» (Bruno Basile, «Introduzione», in Pierio Valeriano, L'infelicità dei letterati, a cura di Bruno Basile, traduzione di Aniello Di Mauro, Napoli, La Scuola di Pitagora, 2010, p. 13). Gli Horti Colocciani, detti anche dell'Acqua Vergine, erano la proprietà suburbana di Angelo Colocci, presso S. Andrea delle Fratte, dove numerosi letterati si riunivano e s'intrattenevano in dotte coversazioni. Per la figura del Colocci si veda la nota 12.

4. Cfr. Valerio Vianello, «Le "bellissime questioni e curiose" nel "Dialogo della Volgar Lingua" di Pierio Valeriano", in Id., Il "giardino" delle parole. Itinerari di scrittura e modelli letterari nel dialogo cinquecentesco, Roma, Jouvence, 1993, pp. 87-109 (a p. 89). 
5. Per l'ed. critica dell'opera si rinvia a Julia Haig Gaisser, Pierio Valeriano on the Ill Fortune of Learned Men. A Renaissance Humanist and His World, Ann Arbor, The University of Michigan Press, 1999, pp. 79-259; ad essa si rifà la più recente edizione curata da Basile (cfr. la nota 3).

6. Il catalogo delle opere del Valeriano si trova in Paolo Pellegrini, Pierio Valeriano e la tipografia del Cinquecento. Nascita, storia e bibliografia delle opere di un umanista, Udine, Forum, 2002.

7. Piero Floriani, «Grammatici e teorici della letteratura volgare», in Storia della cultura veneta, 3 /

II, Vicenza, Neri Pozza, 1980, pp. 139-81 (a p. 141).

8. Vianello, «Le "bellissime questioni e curiose"», cit., p. 91.

9. Cit. da Carlo Dionisotti, Gli umanisti e il volgare fra Quattro e Cinquecento, a cura di Vincenzo Fera, Milano, Continents, 2003 (I ed. Firenze, Le Monnier, 1968), p. 16. Si veda anche la traduzione del 1558 inserita nell'opera etnologica di Giovanni Boemo e citata da Manlio Cortelazzo (I dialetti e la dialettologia in Italia (fino al 1800), Gunter Narr Verlag, Tübingen, 1980, pp. 48-49): «Quello che per l'adietro despiaceva in Roma nella lingua, si acconcia e fa grazioso ogni dì di più per la conversazione di molte genti insieme».

10. Piero Floriani, «La "questione della lingua" e il "dialogo" di P. Valeriano», Giornale Storico della Letteratura Italiana, CLV (1978), n. 491, pp. 321-45 (a p. 341); si veda anche Vianello, «Le "bellissime questioni e curiose"», cit., p. 95. L'esistenza di due diverse redazioni è stata segnalata per la prima volta da Ornella Castellani Pollidori nell'introduzione a Claudio Tolomei, Il Cesano de la lingua toscana, Firenze, Olschki, 1974, pp. 28-31.

11. Vianello, "Le "bellissime questioni e curiose"», cit., pp. 95-107. Per la datazione si vedano anche Carlo Dionisotti, Machiavellerie, Torino, Einaudi, 1980, pp.300-02 e Paolo Trovato, «Introduzione e commento», in Niccolò Machiavelli, Discorso intorno alla nostra lingua, Padova, Antenore, 1982, pp. XXXIV-XXXV e LII.

12. L'Apologia si legge nel volume Rime di Serafino De' Cimminelli Dall'Aquila, a cura di Mario Menghini, Bologna, Romagnoli-Dall'Acqua, 1894, pp. 23-32. Angelo Colocci (1474-1549), esponente di un'antica famiglia di Iesi, nel '98 si trasferì a Roma, dove rivestì varie cariche presso la curia e «divenne una sorta di catalizzatore di esperienze culturali tra le più varie, almeno per un quarantennio» (Giuseppe Frasso, "Appunti su due recenti volumi dedicati a Angelo Colocci», Verbum. Analecta Neolatina, XII/1, 2010, pp. 221-41, a p. 221). Sulle sue idee linguistiche si veda in particolare Nadia Cannata Salamone, «Alle origini della trattatistica sul volgare nel primo Cinquecento: "Le Annotationi sul vulgare ydioma" di Angelo Colocci (ms. Vat. lat. 4831)», The Italinist, 26 (2006), fasc. 2, pp. 197-222; per una panoramica degli studi più recenti sul Colocci si rinvia al succitato articolo di Frasso.

13. Il Colocci compare, accanto allo stesso Pierio e ad altri intellettuali del tempo, anche nel De litteratorum infelicitate.

14. Riccardo Drusi, La lingua cortigiana romana. Note su un aspetto della questione cinquecentesca della lingua, Venezia, Il Cardo, 1995, p. 28.

15. Cfr. Vianello, Le "bellissime questioni e curiose"», cit., p. 94.

16. Floriani sottolinea come tale interpretazione, formulata da Ciro Trabalza nel 1908, sia stata poi condivisa da Benedetto Croce, da Maurizio Vitale e da Ettore Bonora. Cfr. Floriani, «La "questione della lingua" e il "dialogo" di P. Valeriano», cit., p. 341.

17. Cfr. Drusi, La lingua cortigiana romana, cit., pp. 27-31, in particolare p. 29. Al principio del Dialogo, il Colocci racconta che il Trissino «differiva assai alla toscana» (p. 10 della princeps), cioè concedeva molto alla lingua toscana; in queste parole Drusi legge una critica nei confronti della tendenza, propria del vicentino, ad una lingua (anche parlata) eccessivamente toscaneggiante. Ciò sembra però in contrasto con un'affermazione successiva dello stesso Colocci: «il Trissino, molto castigato, né toscaneggiava del tutto, né teneva del venetiano, ma con grave temperamento servendosi dell'uno e dell'altro faceva una soave compositione» (p. 15). Il senso della frase «differiva assai alla [lingua] toscana» si chiarisce meglio se si prende in considerazione 
il contesto in cui si inserisce: un confronto tra la posizione moderata del Trissino e quella radicalmente antitoscana del Tebaldeo.

18. Si ricorda il giudizio di Croce: «Anzitutto, è una scrittura letterariamente briosa e rapida, dice molto in poco e dipinge i personaggi che mette in iscena» (Benedetto Croce, «Pierio Valeriano e la controversia sulla lingua», in Id., Poeti e scrittori del pieno e tardo Rinascimento, vol. II, Bari, Laterza, 1958, pp. 74-84; il passo citato è a p. 75). Si vedano inoltre: Floriani, «La "questione della lingua" e il "dialogo" di P. Valeriano», cit., pp. 340-42; Id., «Grammatici e teorici della letteratura volgare», cit., p. 169; Drusi, La lingua cortigiana romana, cit., p. 27.

19. Si segnalano le due edizioni più recenti del Castellano: Giovan Giorgio Trissino, Scritti linguistici, a cura di Alberto Castelvecchi, Roma, Salerno, 1986, pp. 19-82; Mario Pozzi (a cura di), Discussioni linguistiche del Cinquecento, Torino, UTET, 1988, pp. 105-73.

20. Floriani, «Grammatici e teorici della letteratura volgare», cit., p. 168.

21. Tutte le citazioni sono tratte dall'editio princeps.

22. Il biasimo del Marostica nasce dalla preoccupazione di salvaguardare la naturalezza della libera conversazione tra gli uomini di cultura; al di là delle intenzioni di fondo, simili attestazioni di disagio si riscontrano anche nell'Equicola, nel Castiglione e in autori toscani come l'Aretino e Giambattista Gelli. Cfr. il commento di Pozzi in Discussioni linguistiche del Cinquecento, cit., pp. 50-51, n. 28. Si vedano anche Stefano Gensini, «La lingua cortigiana e i dibattiti linguistici del primo Cinquecento", Bollettino di italianistica, n. s., I (2004), fasc. 2, pp. 93-108 (a p. 101), e Claudio Giovanardi, La teoria cortigiana e il dibattito linguistico nel primo Cinquecento, Roma, Bulzoni, 1998, pp. 50, 100 e 171.

23. A questa affermazione, affidata al Marostica, segue la battuta del Colocci: «Così dev'essere, perché niuno è in corte che si serva delle lettere et erudittion sua con più giudicio del Bembo» (p. 13). Il letterato veneziano viene citato ancora nel prosieguo del Dialogo, come vedremo.

24. Si veda su questo punto l'osservazione di Giovanardi: «L'accenno al Bembo è interessante innanzi tutto perché si stabilisce l'esistenza di una lingua cortigiana parlata, e in secondo luogo perché tale varietà si configura come il registro alto di ogni lingua parlata non toscana. In altri termini, l'uomo del popolo parla la propria "lingua della cuna", mentre il dotto usa la lingua cortigiana» (La teoria cortigiana, cit., p. 154).

25. Ivi, p. 105.

26. I due tratti fonetici descritti rientrano nel generale fenomeno di evoluzione linguistica che caratterizzò il fiorentino del '400: laldando è un caso di reazione ipercorretta alla velarizzazione di $l$ preconsonantica a $u$ (il fenomeno per cui altro > autro); groliosa, forma con metatesi reciproca di $r$ e l, è una variante popolare, documentata già in testi fiorentini e toscani trecenteschi. Per la velarizzazione di $l$ preconsonantica e per altri tratti del fiorentino argenteo si rinvia allo studio ormai classico di Paola Manni, «Ricerche sui tratti fonetici e morfologici del fiorentino quattrocentesco», Studi di grammatica italiana, VII (1979), pp. 115-71.

27. Giovanardi, La teoria cortigiana, cit., p. 143.

28. Cfr. Paolo Trovato, «La questione della lingua e la fissazione della norma», in Id., Il primo Cinquecento, Bologna, il Mulino, 1994, pp. 75-121, a p. 118.

29. Il testo è stato trascritto secondo la copia della Biblioteca Universitaria Alessandrina dell'Università «La Sapienza» di Roma (collocazione: XV f.4 3).

30. Nella stampa: adularli.

31. Nella stampa: contenterà.

32. Nella stampa: moderna.

33. Nella stampa: a.

34. Nella stampa: dalla.

35. Nella stampa: e.

36. Nella stampa: asprete.

37. Nella stampa: saporeto. 
38. Nella stampa: accubinargli.

39. Nella stampa: dano.

\section{RIASSUNTI}

Questo lavoro si inscrive in una serie di schede dedicate alla presentazione sintetica dei principali trattati sul volgare italiano, dal XIV secolo (De Vulgari Eloquentia di Dante Alighieri) alla fine del XVI secolo (Degli avvertimenti della lingua sopra 'l Decamerone di Leonardo Salviati). Ogni scheda comprende una breve presentazione dell'autore, del contesto linguistico e dell'opera, accompagnata da una selezione di estratti significativi e da una bibliografia del corpus e della critica.

\section{INDICE}

indice cronologico XVIe siècle

Mots-clés : Valeriano, lingua italiana, questione della lingua, trattato, 1524-1525, 1529-1530, 1620

Indice geografico : domaine italien

\section{AUTORI}

MARIA LUISA GIORDANO

Maria Luisa Giordano è dottore di ricerca in Storia della lingua e della letteratura italiana e collabora con le cattedre di Linguistica italiana e di Storia della lingua italiana dell'Università degli Studi di Milano (Direttore di Ricerca Prof.ssa Silvia Morgana). 